\title{
FUCOM-MARCOS based Group Decision-making using Dombi Power Aggregation of Dual Probabilistic Linguistic Information
}

Abhijit Saha ( $\nabla$ abhijit84.math@gmail.com )

Techno College of Engineering Agartala

Arunodaya Raj Mishra

Govt. College Jaitwara, Satna

Pratibha Rani

NIT Warangal: National Institute of Technology Warangal

\section{Research Article}

Keywords: Dual probabilistic linguistic term set, Dombi power weighted aggregation operators, FUCOM, MARCOS, multi-criteria group decision making.

Posted Date: August 10th, 2021

DOI: https://doi.org/10.21203/rs.3.rs-371236/v1

License: (c) (i) This work is licensed under a Creative Commons Attribution 4.0 International License. Read Full License 


\title{
FUCOM-MARCOS based group decision-making using Dombi power aggregation of dual probabilistic linguistic information
}

\author{
Abhijit Saha $^{1^{*}}$, Arunodaya Raj Mishra ${ }^{2}$ Pratibha Rani ${ }^{3}$ \\ ${ }^{1}$ Dept. of Mathematics, Techno College of Engineering Agartala, Tripura-799004, India; Email: abhijit84.math@gmail.com \\ ${ }^{2}$ Dept. of Mathematics, Govt. College Jaitwara, Satna, M P, India; Email: arunodaya87@ outlook.com \\ ${ }^{3}$ Dept. of Mathematics, NIT Warangal, Telangana-506004, India; Email: pratibha138@ gmail.com \\ *Correspondence: abhijit84.math@gmail.com
}

\begin{abstract}
:
The dual probabilistic linguistic (DPL) term sets are considered superior to probabilistic linguistic term sets. The power average operator can lessen the effects of the extreme assessing data from some decision-makers with prejudice. Further, the Dombi operators are quite flexible with the general parameter during the aggregation process. Moreover, based on deviation from the maximum consistency by the exclusion of the concern of the redundancy of the comparisons made in criteria pairs, FUCOM (full consistency method) is utilized as a subjective criteria weight computing model. Besides, MARCOS (Measurements alternatives and ranking according to compromise solution) method is based on the determination of utility degrees according to the distance from anti-ideal and ideal solutions and their aggregations. In this study, we combine the merits of power average operator, Dombi operator, FUCOM technique and MARCOS for dealing with multi-criteria group decisionmaking (MCGDM) problems under a DPL setting where rank of the alternatives are obtained through MARCOS method. For aggregating decision-experts preferences, we propose two types of operators, namely- DPL Dombi power weighted averaging and DPL Dombi power weighted geometric aggregation operators. We discuss the elegant properties of these proposed aggregation operators. We provide a case study regarding open source software learning management system selection to focus the practicability and usefulness of the proposed approach. Furthermore, we perform a sensitivity assessment on diverse criteria weight sets in order to test the stability of our developed intriguing approach. To this effect, we also provide a comparison between our approach with various extant methods.
\end{abstract}

Keywords: Dual probabilistic linguistic term set, Dombi power weighted aggregation operators, FUCOM, MARCOS, multi-criteria group decision making.

Table 1: List of abbreviations

\begin{tabular}{|c|c|}
\hline Abbreviation & Full name \\
\hline LT & Linguistic term \\
\hline LTS & Linguistic term set \\
\hline DPL & Dual probabilistic linguistic \\
\hline DPLE & Dual probabilistic linguistic element \\
\hline DPLTS & Dual probabilistic linguistic term set \\
\hline PLTS & Probabilistic linguistic term set \\
\hline MCDM & Multi criteria decision-making \\
\hline MCGDM & Multi criteria group decision-making \\
\hline DE & Decision expert \\
\hline
\end{tabular}




\begin{tabular}{|c|c|}
\hline DM & Decision-making \\
\hline AO & Aggregation operator \\
\hline PAO & Power average operator \\
\hline DPLDPWAA & DPL Dombi power weighted averaging aggregation \\
\hline DPLDPWGA & DPL Dombi power weighted geometric aggregation \\
\hline OSS-LMS & Open source software learning management system \\
\hline WST & Criteria weight set \\
\hline SRCC & Spearman's rank correlation coefficient \\
\hline$\Lambda_{n}$ & Collection of all natural numbers from 1 to $n$ \\
\hline
\end{tabular}

\section{Introduction}

Due to the intricacy of problems and the imprecision of human behavior, it is challenging for decision experts (DEs) to demonstrate decision information as crisp values. Though the evaluation information obtained from DEs is inaccurate or uncertain, DEs cannot give their preferences by providing precise crisp value. In order to effortlessly portray qualitative evaluation information, Herrera and Martinez (2000) presented linguistic term sets (LTSs). Nevertheless, the linguistic variable permits DEs to give decision information using a single linguistic term (LT). Sometimes, DEs cannot amply term their assessments with a single LT, and hesitancy is present among various LTs. For instance, in an assessment of the feasibility of a life insurance policy, a DE may utilize any LT appearing in the LTS, where $S=\left\{\wp_{0}\right.$ : very bad, $\wp_{1}$ : bad, $\wp_{2}$ : moderately bad, $\wp_{3}$ : moderate, $\wp_{4}$ : slightly good, $\wp_{5}$ : good, $\wp_{6}$ : very good $\}$, in order to signify the assessment. If a DE thinks that the policy is good, then it can be characterized as $\left\{\wp_{5}\right\}$. However, due to the difference between DEs' cognitive ability and the complexity of the DM setting, DEs may obtain different assessment values for the same problem in an actual decision-making (DM) problem. In order to evade the shortcoming of the LV, Rodríguez et al. (2012) presented a concept of a hesitant fuzzy linguistic term set (HFLTS), which allows DEs to articulate their preferences for the options with various possible LTs. However, HFLTSs treat all possible assessment values with equal weights or equal importance so as to highlight the hesitation scenario. It is broadly known that, in practical DM problems, the weights of linguistic assessments cannot be ignored, because DEs may have different preference degrees with respect to several possible LTs. The objects of the HFLTS have an equal weight, which is not in accordance with reality. Because DEs offer different LTs, the objects of the HFLTS should have different significance degrees. For instance, if a DE is $70 \%$ sure that a life insurance plan is good, and $30 \%$ is sure that the life insurance plan is moderate, the assessment information contains not only LTs, but also probabilistic information. In order to overcome this problem, Pang et al. (2016) defined probabilistic linguistic term sets (PLTSs) so as to describe LTSs with some possible LTs, as well as associated probabilistic information. PLTSs allow experts to express their respective preferences in a number of different LTs, also providing corresponding probability information for each LT. Liao et al. (2017) developed a programming method with the PLTS for the assessment of hospitals. Zhang and Xing (2017) introduced a modified PLTS-based VIKOR methodology for the evaluation of green suppliers. Kobina et al. (2017) presented various probabilistic linguistic operators for group decision-making (GDM). Furthermore, a new PLTS-based TODIM method was developed by Liu and Teng (2019) in order to evaluate the online products based on reviews in the form of PLTSs. Later, Krishankumar et al. (2018) studied the two-phase probabilistic linguistic preference relation-based DM model. In another study, Krishankumar et al. (2019) extended the classical WASPAS approach under the PLTS context for the assessment of group DM problems. Recently, Liao et al. (2020) have accompanied a survey on PLTSs and their use in MCGDM so as to summarize the analysis on PLTSs. Xu et al. (2020) 
studied an entropy-based approach to DM and applied it to the problem of the selection of a car sharing platform on PLTSs. Ramadass et al. (2020) implemented the integrated PROMETHEE-Borda method for the selection of cloud vendors with a PLTS setting. Liao and Wu (2020) developed the DNMA algorithm for multi-expert multi-criteria decision making problems with PLTSs. Li et al. (2020) provided a multi-stage medical scheme selection process related to referral system under probabilistic linguistic environment. Wang et al. (2021) developed a multi-dimensional methodology with nested PLTSs and applied it to corporate investment. Xue et al. (2021) demonstrated a dynamic reference point method with probabilistic linguistic information based on the regret theory for public health emergency decision-making. Wei et al. (2021) suggested probabilistic linguistic multiple attribute group decision making for location planning of electric vehicle charging stations based on the generalized Dice similarity measures. According to Xie et al. (2017), PLTSs fails to depict the evaluation information from different perspective. To extend the PLTSs, Xie et al. (2017), introduced the notion of dual probabilistic linguistic term set (DPLTS) which can contain two aspects' evaluation information to an alternative under a criterion from the perspective of both belongingness and nonbelongingness grades. They proposed arithmetic AO to deal with MCDM problems with DPLTSs setting. Xie et al. (2020) presented a new MCDM methodology based on the incomplete dual probabilistic linguistic preference relations.

From the comprehensive investigation made above, the following challenges are identified while dealing with dual probabilistic linguistic (DPL) data:

1. The method described by Xie et al. (2017) cannot exclude the impact of extreme assessing criteria values from various biased DEs with diverse preference attitudes.

2. In some realistic issues, it is very clear that all the criteria do not pay equal consideration through the practice. For example, for the post of sales manager, it is bestowed the preference to working experience than the qualification as well as the age of a candidate. Therefore, preference is to be assessed very thoughtfully towards the selection of the appropriate criteria weight. In the existing method (Xie et al. 2017), because of the picking criteria weights arbitrarily by the authors during the final aggregation process, the final ranking order gets influenced. Moreover, the earlier works on DPLTSs (Xie et al. 2017, Xie et al. 2020) cannot tackle a situation when the preference of a relationship among criteria is only known for the determination of criteria weights.

3. Though the algebraic operators (Xie et al. 2017) under DPL information have been utilized for the purpose of aggregation, there is still an urgent need for a more flexible operator which can be adjusted according to the real decision needs.

4. None of the popular ranking methods, namely TOPSIS, COPRAS, TODIM, MAIRCA, MARCOS etc has been utilized so far for the purpose of generating ranking preferences of alternatives. Hence, prioritization of alternatives by extending popular ranking methods under DPL setting is still a challenging issue.

5. Sensitivity analysis of the criteria weights are missing in the literature on DPLTSs.

Motivated by the above challenges and to circumvent them, some contributions made in this paper are:

1. Some flexible operational laws are proposed for DPLTSs by utilizing the features of Dombi operators (Dombi 1982).

2. The DPL-Dombi Power weighted average and geometric AOs are developed to diminish the impacts of outrageous assessing information from some biased DEs during the DM process. These AOs contain a parameter ' $\phi$ ' which gives the flexibility of adjustment according to the real decision needs. 
3. FUCOM (Pamucar et al. 2018a) is used to compute the criteria weights. However, in contrast to the other subjective models (the AHP, the BWM and others), FUCOM gave the smaller deviations of the achieved degrees of the criteria from the most favorable values (Pamucar et al. 2018a).

4. According to Stevic et al. (2019), compare to other MCDM approaches (TOPSIS, COPRAS, TODIM, MAIRCA), MARCOS method is more sensible due to the fusion of results of the ratio approach and reference point sorting approach. Utilizing the benefits of MARCOS method, in this paper, a novel FUCOM-MARCOS methodology is presented by utilizing the aforementioned power weighted AOs to tackle MCGDM problems with DPL data.

5. To elucidate the practicality and usefulness of developed approach, a case study of open source software LMS selection is discussed under DPL environment. A sensitivity investigation is presented to certify the results found by the proposed framework. Lastly, a comparison is presented to validate the superiority of introduced model.

We summarize the remaining paper as below.

In section 2, we present a comprehensive literature review on Dombi operations and MARCOS method. We present some vital concepts related to DPLTSs, Dombi operations and power average operator in section 3. In Section 4, we develop Dombi operational laws for DPL elements (DPLEs) and study their properties. The associated DPL Dombi power AOs such as DPLDPWAA and DPLDPWGA along with their essential properties are also demonstrated. Section 5 deals with a novel FUCOMMARCOS methodology using the proposed power AOs where the criteria values are expressed as DPLEs. In Section 6, a case study of open source software LMS selection is taken to gloss the developed methodology. Section 7 is solely devoted to the discussion of the results obtained. This includes effect of the parameter in ranking results and sensitivity analysis of criteria weights. At the end of this section, a comparative study is employed to affirm the superiority of the developed methodology. In section 8 , some conclusions are added upon the study.

\section{Literature review:}

Here, we give a brief literature review on Dombi operators and MARCOS method.

\subsection{Dombi operators:}

The Dombi operator inaugurated by Dombi (1982), is exceptional as the sign of the parameter discovers whether the operator type is disjunctive or conjunctive and has excellent flexibility with parameters. Liu et al. (2018) proffered MCDM problem utilizing Dombi Bonferroni mean operator on intuitionistic fuzzy sets. He (2018) investigated typhoon disaster assessment by considering Dombi operations in hesitant fuzzy environment. She and Ye (2018) studied some neutrosophic cubic Dombi AOs with applications in decision-making problems. Motivated by Dombi t-conorm and t-norm, a set of single-valued neutrosophic Dombi AOs has been proposed by Wei and Wei (2018). From such existing studies, it is observed that Dombi operational parameters have the advantage of good flexibility with the operational parameter. Jana et al. (2019a) extended the Dombi operator to the picture fuzzy set and proposed the picture fuzzy Dombi AOs. Linguistic picture fuzzy Dombi AOs and their application in decision-making problems have been discussed by Qiyas et al. (2019). Based on operational laws of Dombi intuitionistic fuzzy AOs, Seikh and Mandal (2019) defined a series of AOs under intuitionistic fuzzy environment and utilized to develop a new MAGDM model with uncertain information. Later, Akram et al. (2019) introduced various new Dombi AOs under Pythagorean fuzzy environment with some substantial characteristics like idempotency, monotonicity, boundedness, reducibility, and Commutativity. Motivated by Dombi operators, Jana et al. (2019b) proposed some Dombi AOs for q-rung orthopair fuzzy numbers and discussed their special features. In a further study, 
Jana et al. (2019c, 2020) introduced a series of Bipolar fuzzy Dombi AOs and Bipolar fuzzy Dombi prioritized AOs, respectively, and further applied for MCDM analysis. Based on axiomatic definition of Dombi t-norm and t-conorm, Liu et al. (2020) proposed a series of interval-valued hesitant fuzzy Dombi AOs for information security risk assessment. Further, Ashraf et al. (2020) introduced several Dombi AOs under Spherical fuzzy set context including Spherical fuzzy Dombi weighted averaging, geometric and hybrid AOs and discuss their properties in details. In a recent study, Wu et al. (2020) studied some interval-valued intuitionistic fuzzy Dombi Heronian mean AOs and their application for evaluating the ecological value of forest ecological tourism demonstration areas. However, there is no study regarding the Dombi AOs under DPL environment.

\subsection{MARCOS method}

The MARCOS method originated by Stević et al. (2019), is a new method based on the measurement of alternatives and their ranking in relation to a compromise solution. In this method, the compromise solution includes the determination of utility functions according to the distance from anti-ideal and ideal solutions and their aggregations. Recently, several authors have utilized the MARCOS method for various purposes. For instance, Stević and Brkovic (2020) introduced a hybrid FUCOM-MARCOS method for the evaluation of human resources in a transport company. In a recent study, Ulutaş et al. (2020) proposed an integrated model based on the correlation coefficient and the standard deviation (CCSD), the indifference threshold-based attribute ratio analysis (ITARA) and the MARCOS method. Further, a collective Grey theory-MARCOS model has been studied by Badi and Pamučar (2020) to evaluate and select the best supplier. To improve the robustness of classical MARCOS method, Stanković et al. (2020) studied a novel fuzzy-based MARCOS method for road traffic risk analysis under uncertain environment.

\section{Preliminaries:}

\subsection{Linguistic term set (LTS) and Dual probabilistic linguistic term set (DPLTS)}

Definition 1 (Zadeh 1975): A discrete LTS $\Gamma=\left\{\wp_{\alpha}: \alpha=-\tau, \ldots, 0, \ldots ., \tau\right\}$ is a set $\left(\wp_{\alpha}\right.$ denotes a linguistic variable and $\tau$ denotes a positive integer) which satisfies the following conditions:

1. $\operatorname{neg}\left(\wp_{\alpha}\right)=\wp_{\delta}$ where $\alpha+\delta=2 \tau$.

2. $\Gamma$ is an ordered set i.e; $\wp_{\alpha} \leq \wp_{\varsigma}$ if $\alpha \leq \varsigma$.

Based on the LST $\Gamma$, Gou et al. (2017) introduced two reversible transformation functions $\tilde{g}:[-\tau, \tau] \rightarrow[0,1]$ and $\tilde{g}^{-1}:[0,1] \rightarrow[-\tau, \tau]$, respectively which are defined as:

$$
\begin{aligned}
& \tilde{g}\left(\wp_{\alpha}\right)=\frac{\alpha}{2 \tau}+\frac{1}{2}(\alpha=-\tau, \ldots, 0, \ldots, \tau) \\
& \tilde{g}^{-1}(\beta)=\wp_{(2 \beta-1) \tau}(\beta \in[0,1])
\end{aligned}
$$

Definition 2 (Xie et al. 2017): Let $U=\left\{x_{1}, x_{2}, \ldots \ldots ., x_{n}\right\}$ denotes a fixed set and $\Gamma=\left\{\wp_{\alpha}: \alpha=-\tau, \ldots, 0, \ldots ., \tau\right\}$ be a LST. Then a DPLTS $Y_{\wp}(\mathcal{Z})$ on $U$ is given by

$$
Y_{\wp}(\boldsymbol{Z})=\left\{<x_{i}, \mu_{\wp}(\boldsymbol{Z})\left(x_{i}\right), \gamma_{\wp}(\boldsymbol{Z})\left(x_{i}\right)>: x_{i} \in U\right\}
$$

where $\quad \mu_{\wp}(\boldsymbol{Z})\left(x_{i}\right)=\left\{\wp_{\xi^{i(u)}}\left(\boldsymbol{J}^{(u)}\right): \wp_{\xi^{i}(u)} \in \Gamma, \boldsymbol{J}^{(u)} \geq 0, \sum_{u=1}^{T} \boldsymbol{J}^{(u)} \leq 1\right\} \quad$ and $\quad \gamma_{\wp}(\boldsymbol{Z})\left(x_{i}\right)=\left\{\wp_{g^{i(v)}}\left(\boldsymbol{J}^{(v)}\right): \wp_{g^{i(v)}} \in \Gamma\right.$,

$\left.\boldsymbol{\mho}^{(v)} \geq 0, \sum_{v=1}^{R} \boldsymbol{\mho}^{(v)} \leq 1\right\}$ represent the linguistic belongingness and non-belongingness grade respectively of 
$x_{i} \in U$ and the associated probabilities are $\mho^{(u)}$ and $\mho^{(v)}$ respectively; $\xi^{i(u)}$ and $\vartheta^{i(v)}$ are the subscripts of the LTs $\wp_{\xi^{i(u)}}$ and $\wp_{g^{i(v)}}$ respectively.

If the set $Y_{\wp}(\widetilde{J)}$ contains only one element, then it reduces to a DPL element (DPLE) and we denote it $\quad$ as: $\quad y_{\wp}(\widetilde{\delta})=<\left\{\wp_{\xi^{i(u)}}\left(\delta^{(u)}\right)\right\},\left\{\wp_{g^{i(v)}}\left(\widetilde{\delta}^{(v)}\right)\right\}>\quad$ where $\quad \wp_{\xi^{i(u)}}, \wp_{\xi^{i(v)}} \in \Gamma \quad$ and $\boldsymbol{\mho}^{(u)}, \boldsymbol{\mho}^{(v)} \geq 0, \sum_{u=1}^{T} \boldsymbol{\mho}^{(u)} \leq 1, \sum_{v=1}^{R} \boldsymbol{\mho}^{(v)} \leq 1$.

Definition 3 (Xie et al. 2017): Let $y_{\wp}^{(j)}(\widetilde{\delta})=<\left\{\wp_{\xi^{j(u)}}\left(\boldsymbol{\mho}^{j(u)}\right)\right\},\left\{\wp_{\xi^{j(v)}}\left(\boldsymbol{J}^{j(v)}\right)\right\}>(j=1,2)$ be two DPLEs, where $u \in \Lambda_{T}, v \in \Lambda_{R}$. For sake of simplicity, let us take, $\tilde{g}_{\xi}^{j}=\tilde{g}\left(\wp_{\xi^{j(u)}}\right)$ and $\tilde{g}_{\vartheta}^{j}=\tilde{g}\left(\wp_{g^{j(v)}}\right)(j=1,2)$. Then, the basic operations of DPLEs are as follows:

(i) $\operatorname{Neg}\left(\tilde{y}_{\wp}^{(1)}(\boldsymbol{\sigma})\right)=\left\langle\left\{\wp_{\xi^{1(v)}}\left(\boldsymbol{J}^{1(v)}\right)\right\},\left\{\wp_{\xi^{(u)}}\left(\boldsymbol{J}^{1(u)}\right)\right\}\right\rangle$

(ii) $\tilde{y}_{\xi}^{(1)}(\boldsymbol{W}) \oplus \tilde{y}_{\xi}^{(2)}(\boldsymbol{J})=\left\langle\left\{\tilde{g}^{-1}\left(\tilde{g}_{\xi}^{1}+\tilde{g}_{\xi}^{2}-\tilde{g}_{\xi}^{1} \tilde{g}_{\xi}^{2}\right)\left(\boldsymbol{J}^{1(u)} \boldsymbol{W}^{2(u)}\right)\right\}, \tilde{g}^{-1}\left(\tilde{g}_{\vartheta}^{1} \tilde{g}_{\vartheta}^{2}\right)\left(\boldsymbol{J}^{1(v)} \boldsymbol{J}^{2(v)}\right)\right\rangle$

(iii) $\tilde{y}_{\wp}^{(1)}(\widetilde{\mho}) \otimes \tilde{y}_{\wp}^{(2)}(\widetilde{\mho})=\left\langle\tilde{g}^{-1}\left(\tilde{g}_{\xi}^{1} \tilde{g}_{\xi}^{2}\right)\left(\widetilde{\mho}^{1(u)} \boldsymbol{\mho}^{2(u)}\right),\left\{\left\{\tilde{g}^{-1}\left(\tilde{g}_{\vartheta}^{1}+\tilde{g}_{\vartheta}^{2}-\tilde{g}_{\vartheta}^{1} \tilde{g}_{\vartheta}^{2}\right)\left(\boldsymbol{\mho}^{1(v)} \boldsymbol{\mho}^{2(v)}\right)\right\}\right\rangle\right.$

(iv) $\lambda \tilde{y}_{\wp}^{(1)}(\widetilde{\delta})=\left\langle\left\{\tilde{g}^{-1}\left(1-\left(1-\tilde{g}_{\xi}^{1}\right)^{\lambda}\right)\left(\boldsymbol{J}^{1(u)}\right)\right\}, \tilde{g}^{-1}\left(\left(\tilde{g}_{\vartheta}^{1}\right)^{\lambda}\right)\left(\boldsymbol{\delta}^{1(v)}\right)\right\rangle$

(v) $\left(\tilde{y}_{\wp}^{(1)}(\widetilde{\delta})\right)^{\lambda}=\left\langle\tilde{g}^{-1}\left(\left(\tilde{g}_{\xi}^{1}\right)^{\lambda}\right)\left(\boldsymbol{J}^{1(u)}\right),\left\{\tilde{g}^{-1}\left(1-\left(1-\tilde{g}_{\vartheta}^{1}\right)^{\lambda}\right)\left(\boldsymbol{\sigma}^{1(v)}\right)\right\}\right\rangle$

Theorem 1: Let $y_{\wp}^{(j)}(\widetilde{\zeta})=<\left\{\wp_{\xi^{j(u)}}\left(\boldsymbol{J}^{j(u)}\right)\right\},\left\{\wp_{g^{j(v)}}\left(\boldsymbol{J}^{j(v)}\right)\right\}>(j=1,2)$ be two DPLEs, where $u \in \Lambda_{T}, v \in \Lambda_{R}$. Then, for any $\lambda, \lambda_{1}, \lambda_{2}>0$, we have,

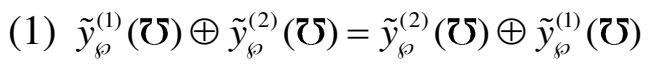

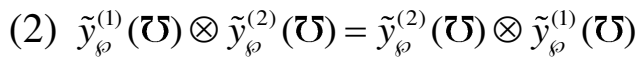

(3) $\lambda\left(\tilde{y}_{\wp}^{(1)}(\widetilde{\delta}) \oplus \tilde{y}_{\wp}^{(2)}(\widetilde{\delta})\right)=\left(\lambda \tilde{y}_{\wp}^{(1)}(\widetilde{\delta})\right) \oplus\left(\lambda \tilde{y}_{\wp}^{(2)}(\widetilde{\delta})\right)$

(4) $\left(\tilde{y}_{\wp}^{(1)}\left(\widetilde{J)} \otimes \tilde{y}_{\wp}^{(2)}(\widetilde{\delta})\right)^{\lambda}=\left(\tilde{y}_{\wp}^{(1)}(\widetilde{J})\right)^{\lambda} \otimes\left(\tilde{y}_{\wp}^{(2)}(\widetilde{J})\right)^{\lambda}\right.$

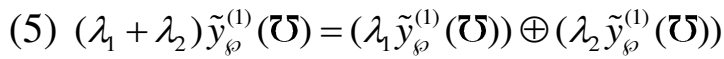

(6) $\left(\tilde{y}_{\wp}^{(1)}(\widetilde{J})\right)^{\lambda_{1}+\lambda_{2}}=\left(\tilde{y}_{\wp}^{(1)}(\widetilde{J})\right)^{\lambda_{1}} \otimes\left(\tilde{y}_{\wp}^{(1)}(\widetilde{J})\right)^{\lambda_{2}}$

Proof: Straight forward.

Xie et al. (2017) defined the terms- score value and accuracy value of a DPLE. But the formulas' they used have very complex mathematical structures. In order to simplify those, we introduce them as stated below.

Definition 4: Let $y_{\wp}(\widetilde{\mho})=<\mu_{\wp}(\widetilde{\mho}), \gamma_{\wp}(\widetilde{Z})>=<\left\{\wp_{\xi^{(u)}}\left(\widetilde{J}^{(u)}\right)\right\},\left\{\wp_{g^{(v)}}\left(\widetilde{J}^{(v)}\right)\right\}>$ be a DPLE where $u \in \Lambda_{T}, v \in \Lambda_{R}$ . Then the score value of $y_{\wp}(\widetilde{\delta})$ is given by

$$
S\left(y_{\wp}(\widetilde{\zeta)})=\sum_{u=1}^{\# T_{\xi}} \tilde{g}\left(\wp_{\xi^{(u)}}\right)\left(\boldsymbol{\mho}^{(u)}\right)-\sum_{v=1}^{\# T_{\vartheta}} \tilde{g}\left(\wp_{g^{(v)}}\right)\left(\boldsymbol{\mho}^{(v)}\right)\right.
$$


where $\tilde{g}\left(\wp_{\xi^{(u)}}\right), \tilde{g}\left(\wp_{g^{(v)}}\right) \in[0,1], \# T_{\xi}$ and $\# T_{\vartheta}$ signify the number of objects in $\mu_{\wp}(\mho)$ and $\gamma_{\wp}(\mho)$ respectively.

The greater value of $S\left(y_{\wp}(\mho)\right)$, the better DPLEs will be. But it is not sufficient to compare the DPLEs with the same score values. To handle this type of cases, let us introduce the notion of accuracy value given as:

Definition 5: Let $y_{\wp}(\widetilde{\mho})=<\mu_{\wp}(\widetilde{\mho}), \gamma_{\wp}(\widetilde{\mho})>=<\left\{\wp_{\xi^{(u)}}\left(\mho^{(u)}\right)\right\},\left\{\wp_{g^{(v)}}\left(\mho^{(v)}\right)\right\}>$ be a DPLE where $u \in \Lambda_{T}, v \in \Lambda_{R}$ . Then the accuracy value of $y_{\wp}(\mho)$ is given by:

$$
A\left(y_{\wp}(\widetilde{\delta})\right)=\sum_{u=1}^{\# T_{\xi}} \tilde{g}\left(\wp_{\xi^{(u)}}\right)\left(\boldsymbol{\mho}^{(u)}\right)+\sum_{v=1}^{\# T_{g}} \tilde{g}\left(\wp_{\vartheta^{(v)}}\right)\left(\boldsymbol{\mho}^{(v)}\right)
$$

where $\tilde{g}\left(\wp_{\xi^{(u)}}\right), \tilde{g}\left(\wp_{g^{(v)}}\right) \in[0,1], \# T_{\xi}$ and $\# T_{\vartheta}$ signify the number of objects in the adjusted sets $\mu_{\wp}(\mho)$ and $\gamma_{\wp}(\mho)$, respectively.

Definition 6 (Xie et al. 2017): Let $y_{\wp}^{(1)}\left(\widetilde{S)}\right.$ and $y_{\wp}^{(2)}(\widetilde{\delta})$ be two DPLEs. Then, a comparative structure for DPLEs can be described as

A. If $S\left(y_{\wp}^{(1)}(\widetilde{\delta})\right)>S\left(y_{\wp}^{(2)}(\widetilde{\Xi})\right)$, then $y_{\wp}^{(1)}(\widetilde{\Xi}) \succ y_{\wp}^{(2)}(\widetilde{\mho})$

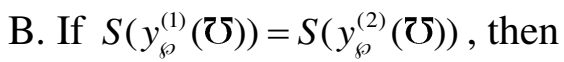

(a) If $A\left(y_{\wp}^{(1)}(\widetilde{\mho})\right)>A\left(y_{\wp}^{(2)}(\widetilde{\mho})\right)$, then $y_{\wp}^{(1)}(\widetilde{\mho}) \succ y_{\wp}^{(2)}(\widetilde{\mho})$

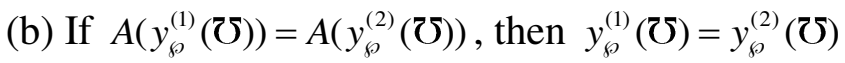

\subsection{Dombi operations}

The operations of $t$-norm and $t$-conorm, developed by Dombi (1982) are generally known as Dombi operations described below:

Definition 7: (Dombi 1982) For any two real numbers $x$ and $y$ in $[0,1]$, the Dombi $t$-norm and Dombi $t$-conorm can be defined as follows:

$$
\operatorname{Dom}(x, y)=\frac{1}{1+\left\{\left(\frac{1-x}{x}\right)^{\phi}+\left(\frac{1-y}{y}\right)^{\phi}\right\}^{\frac{1}{\phi}}}, \operatorname{Dom}^{c}(x, y)=1-\frac{1}{1+\left\{\left(\frac{x}{1-x}\right)^{\phi}+\left(\frac{y}{1-y}\right)^{\phi}\right\}^{\frac{1}{\phi}}}(\phi \geq 1)
$$

Dombi operations have good precedence of change w.r.t values of the parameter ' $\phi$ '.

\subsection{Power average operator (PAO)}

Definition 8 (Yager 2001): Let $u_{1}, u_{2}, \ldots \ldots ., u_{n}$ are considered as accumulation of crisp numbers. Then the power average (PA) operator associated to aggregation of these numbers is given by

$$
P A\left(u_{1}, u_{2}, \ldots, u_{n}\right)=\frac{\sum_{i=1}^{n}\left(1+\Delta\left(u_{i}\right)\right) u_{i}}{\sum_{i=1}^{n}\left(1+\Delta\left(u_{i}\right)\right)} \quad \text { where } \Delta\left(u_{i}\right)=\sum_{j=1, j \neq i}^{n} \operatorname{Supp}\left(u_{i}, u_{j}\right)
$$


Here, $\operatorname{Supp}\left(u_{i}, u_{j}\right)$ denotes the support for $u_{i}$ from $u_{j}$ and has three postulates as

(i) $0 \leq \operatorname{Supp}\left(u_{i}, u_{j}\right) \leq 1$

(ii) $\operatorname{Supp}\left(u_{i}, u_{j}\right)=\operatorname{Supp}\left(u_{j}, u_{i}\right)$

(iii) $\operatorname{Supp}\left(u_{i}, u_{j}\right) \geq \operatorname{Supp}\left(u_{k}, u_{r}\right)$ provided $\left|u_{i}-u_{j}\right|<\left|u_{k}-u_{r}\right|$ where $i, j, k, r \in N_{n}$.

\section{Dombi operations and Dombi power weighed aggregation operators}

For the given DPLEs $y_{\wp}^{(1)}(\widetilde{\delta})$ and $y_{\wp}^{(2)}(\widetilde{\delta})$, sometimes the probability and the LTs in $y_{\wp}^{(1)}(\widetilde{\delta})$ and $y_{\wp}^{(2)}(\widetilde{\mho})$ becomes different. Multiplication of the probabilities with their corresponding linguistic indices may lead to unreasonable conclusions in process of information aggregation. To address this issue, we apply the method of adjustment of probabilities the procedure of which is described in the following example.

Example 1: Let $\Gamma=\left\{\wp_{\tau}: \tau=-3,-2,-1,0,1,2,3\right\}$ be a discrete LTS. Take two DPLEs $y_{\wp}^{(1)}(\mho)=<\left\{\wp_{2}(0.8), \wp_{3}(0.2)\right\},\left\{\wp_{-1}(0.5), \wp_{-2}(0.5)\right\}>$ and $y_{\wp}^{(2)}(\mho)=<\left\{\wp_{0}(1)\right\},\left\{\wp_{2}(0.4), \wp_{3}(0.6)\right\}>$. Then their corresponding adjusted DPLEs are: $\tilde{y}_{\wp}^{(1)}(\widetilde{\mho})=<\left\{\wp_{2}(0.8), \wp_{3}(0.2)\right\},\left\{\wp_{-1}(0.4), \wp_{-1}(0.1), \wp_{-2}(0.5)\right\}>$ and $\tilde{y}_{\wp}^{(2)}(\widetilde{\mho})=<\left\{\wp_{0}(0.8), \wp_{0}(0.2)\right\},\left\{\wp_{2}(0.4), \wp_{3}(0.1), \wp_{3}(0.5)\right\}>$. The adjustment procedure is shown below (Fig. $1)$.

\begin{tabular}{|c|c|c|c|c|}
\hline \multirow[b]{2}{*}{ Membership degree (original): } & \multicolumn{2}{|c|}{$\underline{y_{\wp}^{(1)}(\widetilde{O})}$} & $\underline{y_{\wp}^{(2)}(\widetilde{)})}$ & \\
\hline & $\begin{array}{c}\wp_{2}(0.8) \\
\downarrow\end{array}$ & $\begin{array}{c}\wp_{3}(0.2) \\
\downarrow\end{array}$ & $\wp_{0}(1)$ & \\
\hline Membership degree (adjusted): & $\wp_{2}(0.8)$ & $\wp_{3}(0.2)$ & $\wp_{0}(0.8)$ & $\wp_{0}(0.2)$ \\
\hline Non-membership degree (original): & $\wp_{-1}(0.5)$ & $\wp_{-2}(0.5)$ & $\wp_{2}(0.4)$ & $\wp_{3}(0.6)$ \\
\hline & $\swarrow \searrow$ & $\downarrow$ & $\downarrow$ & $\swarrow \searrow$ \\
\hline Non-membership degree (adjusted): & ${ }_{-1}(0.4) \wp_{-1}$ & $\wp_{-2}(0.5)$ & $\wp_{2}(0.4)$ & $\wp_{3}(0.1) \wp_{3}(0.5)$ \\
\hline
\end{tabular}

Fig. 1: Adjustment procedure of DPLEs

Definition 9: Let $\tilde{y}_{\wp}^{(j)}\left(\widetilde{\zeta)}=<\left\{\wp_{\xi^{j(u)}}\left(\widetilde{J}^{(u)}\right)\right\},\left\{\wp_{g^{j(v)}}\left(\widetilde{J}^{(v)}\right)\right\}>(j=1,2)\right.$ be two adjusted DPLEs where $u \in \Lambda_{T}, v \in \Lambda_{R}$ and $\lambda>0$. Then we define the Dombi operations between DPLEs as follows:

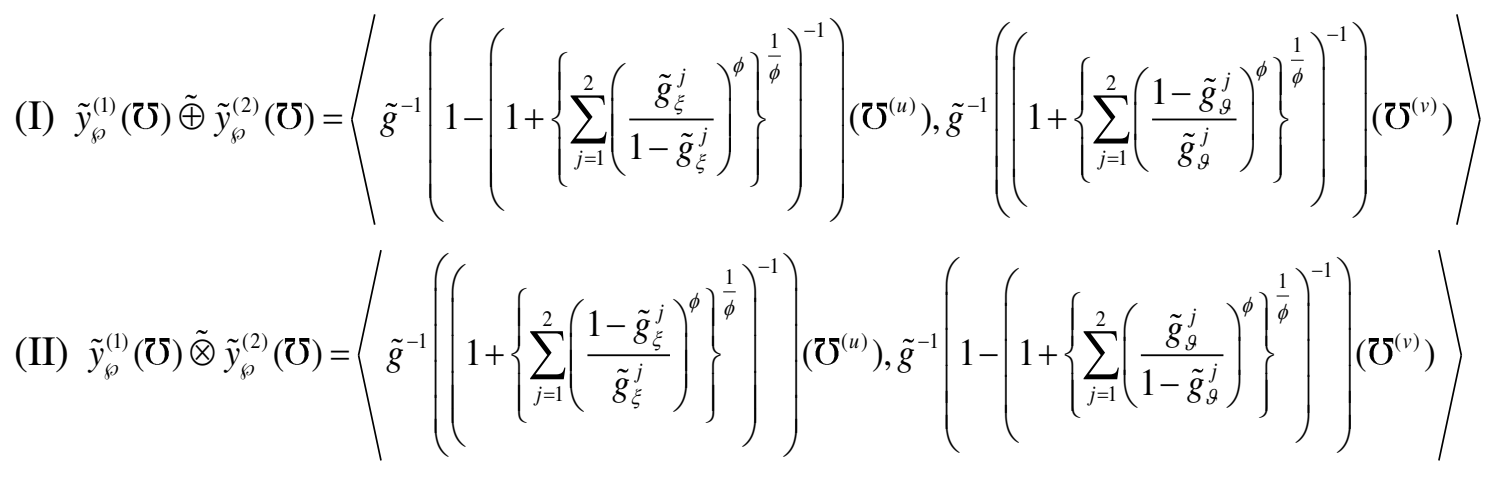


(III) $\left.\lambda \tilde{y}_{\xi}^{(1)}(\widetilde{\sigma})=\left\langle\tilde{g}^{-1}\left(1-\left(1+\left\{\lambda\left(\frac{\tilde{g}_{\xi}^{1}}{1-\tilde{g}_{\xi}^{1}}\right)^{\phi}\right\}^{\frac{1}{\phi}}\right\}^{-1}\right)\left(\widetilde{\mho}^{(u)}\right), \tilde{g}^{-1}\left(1+\left\{\lambda\left(\frac{1-\tilde{g}_{9}^{1}}{\tilde{g}_{9}^{1}}\right)^{\phi}\right\}^{\frac{1}{\phi}}\right)^{-1}\right)\left(\widetilde{J}^{(\omega)}\right)\right\rangle$

(IV) $\left(\tilde{y}_{\wp}^{(1)}(\widetilde{\mho})\right)^{\lambda}=\left\langle\tilde{g}^{-1}\left(\left(1+\left\{\lambda\left(\frac{1-\tilde{g}_{\xi}^{1}}{\tilde{g}_{\xi}^{1}}\right)^{\phi}\right\}^{\frac{1}{\phi}}\right)^{-1}\right)\left(\widetilde{\mho}^{(u)}\right), \tilde{g}^{-1}\left(1-\left(1+\left\{\lambda\left(\frac{\tilde{g}_{\vartheta}^{1}}{1-\tilde{g}_{\vartheta}^{1}}\right)^{\phi}\right\}^{\frac{1}{\phi}}\right)^{-1}\right)\left(\widetilde{J}^{(v)}\right)\right\rangle$

Theorem 2: Let $\tilde{y}_{\wp^{\prime}}^{(j)}(\widetilde{\delta})=<\left\{\wp_{\xi^{j(u)}}\left(\mho^{(u)}\right)\right\},\left\{\wp_{g^{j(v)}}\left(\mho^{(v)}\right)\right\}>(j=1,2)$ be two adjusted DPLEs where $u \in \Lambda_{T}, v \in \Lambda_{R}$. Then, for any $\lambda, \lambda_{1}, \lambda_{2}>0$, we have,

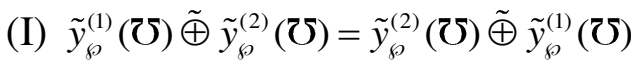

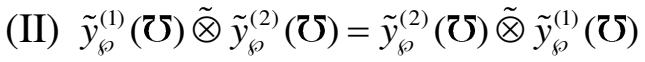

(III) $\lambda\left(\tilde{y}_{\wp}^{(1)}(\widetilde{\Psi}) \tilde{\oplus} \tilde{y}_{\wp}^{(2)}(\widetilde{J})\right)=\left(\lambda \tilde{y}_{\wp}^{(1)}(\widetilde{J})\right) \tilde{\oplus}\left(\lambda \tilde{y}_{\wp}^{(2)}(\widetilde{J})\right)$

(IV) $\left(\tilde{y}_{\wp}^{(1)}\left(\widetilde{\Xi)} \tilde{\otimes} \tilde{y}_{\wp}^{(2)}(\widetilde{J})\right)^{\lambda}=\left(\tilde{y}_{\wp}^{(1)}(\widetilde{J})\right)^{\lambda} \tilde{\otimes}\left(\tilde{y}_{\wp}^{(2)}(\widetilde{J})\right)^{\lambda}\right.$

(V) $\left(\lambda_{1}+\lambda_{2}\right) \tilde{y}_{\wp}^{(1)}(\widetilde{\mho})=\left(\lambda_{1} \tilde{y}_{\wp}^{(1)}(\widetilde{\Psi})\right) \tilde{\oplus}\left(\lambda_{2} \tilde{y}_{\wp}^{(1)}(\widetilde{J})\right)$

(VI) $\left(\tilde{y}_{\wp}^{(1)}(\widetilde{\zeta})\right)^{\lambda_{1}+\lambda_{2}}=\left(\tilde{y}_{\wp}^{(1)}(\widetilde{\mho})\right)^{\lambda_{1}} \tilde{\otimes}\left(\tilde{y}_{\wp}^{(1)}(\widetilde{\zeta})\right)^{\lambda_{2}}$

Proof: Added in supplementary material.

\subsection{DPL Dombi Power weighted AOs}

This sub-section is dedicated to the development of the operators DPLDPWAA and DPLDPWGA.

\subsubsection{DPL Dombi power weighted averaging AO}

Definition 10: Suppose $\tilde{y}_{\xi}^{(j)}(\widetilde{\zeta})=<\left\{\wp_{\xi^{j(u)}}\left(\widetilde{\mho}^{(u)}\right)\right\},\left\{\wp_{g^{j(v)}}\left(\widetilde{\mho}^{(v)}\right)\right\}>\left(j \in \Lambda_{n}\right)$ be a collection of adjusted DPLEs $\left(u \in \Lambda_{T} ; v \in \Lambda_{R}\right)$. Then the DPLDPWAA operator is defined as follows:

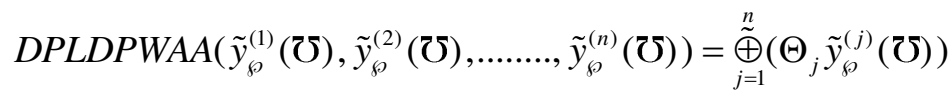

$$
\begin{aligned}
& \text { Here } \Theta_{j}=\frac{\left(1+\sum_{i=1, j \neq i}^{n} \operatorname{Supp}\left(\tilde{y}_{\wp}^{(i)}(\boldsymbol{\mho}), \tilde{y}_{\wp}^{(j)}(\boldsymbol{\mho})\right)\right) w_{j}}{\sum_{j=1}^{n} w_{j}\left(1+\sum_{i=1, j \neq i}^{n} \operatorname{Supp}\left(\tilde{y}_{\wp}^{(i)}(\boldsymbol{\mho}), \tilde{y}_{\wp}^{(j)}(\boldsymbol{\mho})\right)\right)}
\end{aligned}
$$

where $w_{j}(>0)$ is the weight of $\tilde{y}_{\wp}^{(j)}(\boldsymbol{\delta})$ such that $\sum_{j=1}^{n} w_{j}=1$,

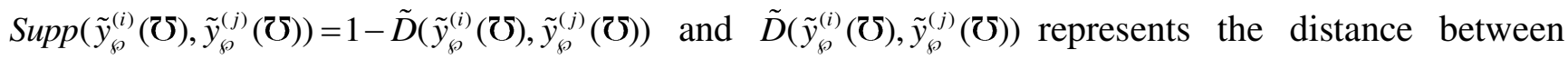
$\tilde{y}_{\wp}^{(i)}\left(\widetilde{\zeta)}\right.$ and $\tilde{y}_{\wp}^{(j)}(\widetilde{\zeta)}$.

Theorem 3: Suppose $\tilde{y}_{\wp}^{(j)}(\widetilde{\delta})=<\left\{\wp_{\xi^{j(u)}}\left(\mathcal{J}^{(u)}\right)\right\},\left\{\wp_{g^{(v)}}\left(\mathcal{J}^{(v)}\right)\right\}>\left(j \in \Lambda_{n}\right)$ be a collection of adjusted DPLEs $\left(u \in \Lambda_{T} ; v \in \Lambda_{R}\right)$. Then the aggregated value is again a DPLE and 


$$
\begin{aligned}
& \operatorname{DPLDPWAA}\left(\tilde{y}_{\wp}^{(1)}(\widetilde{\mho}), \tilde{y}_{\wp}^{(2)}(\widetilde{\mho}), \ldots \ldots \ldots, \tilde{y}_{\wp}^{(n)}(\widetilde{\mho})\right) \\
& \left.=\left\langle\tilde{g}^{-1}\left(1-\left(1+\left\{\sum_{j=1}^{n} \Theta_{j}\left(\frac{\tilde{g}_{\xi}^{j}}{1-\tilde{g}_{\xi}^{j}}\right)^{\phi}\right\}^{\frac{1}{\phi}}\right)^{-1}\right)\left(\mho^{(u)}\right), \tilde{g}^{-1}\left(1+\left\{\sum_{j=1}^{n} \Theta_{j}\left(\frac{1-\tilde{g}_{\vartheta}^{j}}{\tilde{g}_{\vartheta}^{j}}\right)^{\phi}\right\}^{\frac{1}{\phi}}\right)^{-1}\right)\left(\mho^{(v)}\right)\right\rangle
\end{aligned}
$$

where $w_{j}(>0)$ is the weight of $\tilde{y}_{\wp}^{(j)}(\widetilde{\mho})$ such that $\sum_{j=1}^{n} w_{j}=1$.

Proof: Added in the supplementary material.

Theorem 4: (Idempotency) Suppose $\tilde{y}_{\wp}^{(j)}\left(\widetilde{\zeta)}=<\left\{\wp_{\xi^{j(u)}}\left(\widetilde{J}^{(u)}\right)\right\},\left\{\wp_{g^{j(v)}}\left(\widetilde{J}^{(v)}\right)\right\}>\left(j \in \Lambda_{n}\right)\right.$ be a collection of

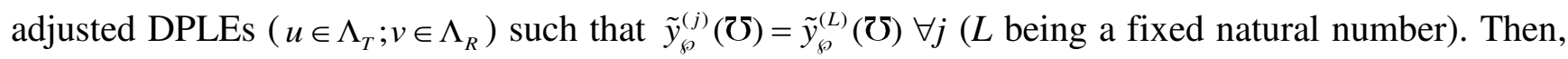
we have $\operatorname{DPLDPWAA}\left(\tilde{y}_{\wp}^{(1)}(\widetilde{\delta}), \tilde{y}_{\wp}^{(2)}(\widetilde{\zeta}), \ldots, \tilde{y}_{\wp}^{(n)}(\widetilde{\zeta})\right)=\tilde{y}_{\wp}^{(L)}(\widetilde{\zeta})$.

Proof: Added in the supplementary material.

Theorem 5: (Monotonicity) Let $\tilde{y}_{\wp}^{(j)}(\widetilde{\delta})=<\left\{\wp_{\xi^{j(u)}}\left(\widetilde{J}^{(u)}\right)\right\},\left\{\wp_{g^{j(v)}}\left(\widetilde{J}^{(v)}\right)\right\}>$ and $\tilde{y}_{\wp}^{(j)}(\widetilde{\zeta})=<\left\{\wp_{\xi^{j(u)}}^{\prime}\left(\widetilde{J}^{(u)}\right)\right\}$, $\left\{\wp_{y^{j(v)}}^{\prime}\left(\widetilde{\mho}^{(v)}\right)\right\}>\left(j \in \Lambda_{n}\right)$ be two collection of adjusted DPLEs $\left(u \in \Lambda_{T} ; v \in \Lambda_{R}\right)$ such that $\forall j, \wp_{\xi^{j(u)}} \leq \wp_{\xi^{j(u)}}^{\prime}$ and $\quad \wp_{g^{j(v)}} \geq \wp_{y^{j(v)}}^{\prime} \quad$ Then, $\quad \operatorname{DPLDPWAA}\left(\tilde{y}_{\wp}^{(1)}(\widetilde{\mho}), \tilde{y}_{\wp}^{(2)}(\widetilde{\mho}), \ldots, \tilde{y}_{\wp}^{(n)}(\widetilde{\mho})\right) \leq D P L D P W A A$ $\left(\tilde{y}_{\wp}^{\prime(1)}(\widetilde{\zeta}), \tilde{y}_{\wp}^{\prime(2)}(\widetilde{\zeta}), \ldots \ldots, \tilde{y}_{\wp}^{\prime(n)}(\widetilde{Z})\right)$.

Proof: Added in the supplementary material.

Theorem 6: (Boundedness) Suppose $\tilde{y}_{\wp}^{(j)}(\widetilde{\delta})=<\left\{\wp_{\xi^{j(u)}}\left(\widetilde{\delta}^{(u)}\right)\right\},\left\{\wp_{g^{(v)}}\left(\widetilde{J}^{(v)}\right)\right\}>\left(j \in \Lambda_{n}\right)$ be a collection of adjusted DPLEs. If $\quad \tilde{y}_{\wp}^{(j-)}(\widetilde{\zeta})=<\left\{\min _{u=1}^{T} \wp_{\xi^{j(u)}}\left(\widetilde{J}^{(u)}\right)\right\},\left\{\max _{v=1}^{R} \wp_{\xi^{j(v)}}\left(\widetilde{J}^{(v)}\right)\right\}>\quad$ and

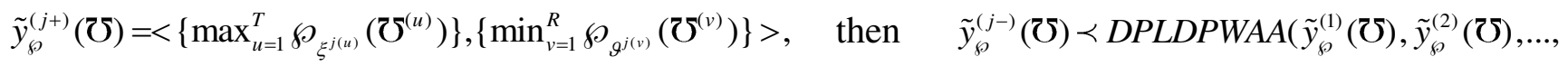
$\tilde{y}_{\wp}^{(n)}(\widetilde{\zeta)}) \prec \tilde{y}_{\wp}^{(j+)}(\widetilde{\delta})$.

Proof: Similar to theorem 5.

\subsubsection{DPL Dombi power weighted geometric AO}

Definition 11: Suppose $\tilde{y}_{\wp}^{(j)}(\widetilde{\mho})=<\left\{\wp_{\xi^{j(u)}}\left(\widetilde{\mho}^{(u)}\right)\right\},\left\{\wp_{g^{j(v)}}\left(\widetilde{\mho}^{(v)}\right)\right\}>\left(j \in \Lambda_{n}\right)$ be a collection of adjusted DPLEs where $u \in \Lambda_{T} ; v \in \Lambda_{R}$. Then the DPLDPWGA operator is defined as follows:

$$
\operatorname{DPLDPWGA}\left(\tilde{y}_{\wp}^{(1)}(\boldsymbol{\delta}), \tilde{y}_{\wp}^{(2)}(\boldsymbol{\delta}), \ldots, \tilde{y}_{\wp}^{(n)}(\boldsymbol{\delta})\right)=\tilde{\bigotimes}_{j=1}^{n}\left(\tilde{y}_{\wp}^{(j)}(\bar{\delta})\right)^{\Theta_{j}}
$$

where $w_{j}(>0)$ is the weight of $\tilde{y}_{\wp}^{(j)}(\mho)$ such that $\sum_{j=1}^{n} w_{j}=1$, and $\Theta_{j}$ is given by Eq. (9).

Theorem 7: Suppose $\tilde{y}_{\wp}^{(j)}(\widetilde{\mho})=<\left\{\wp_{\xi^{j(u)}}\left(\widetilde{\mho}^{(u)}\right)\right\},\left\{\wp_{g^{j(v)}}\left(\widetilde{\mho}^{(v)}\right)\right\}>\left(j \in \Lambda_{n}\right)$ be a collection of adjusted DPLEs where $u \in \Lambda_{T} ; v \in \Lambda_{R}$. Then the aggregated value is again a DPLE and 


$$
\begin{aligned}
& \operatorname{DPLDPWGA}\left(\tilde{y}_{\xi}^{(1)}(\widetilde{\mho}), \tilde{y}_{\xi}^{(2)}(\widetilde{\mho}), \ldots \ldots \ldots, \tilde{y}_{\wp}^{(n)}(\widetilde{\mho})\right) \\
& =\left\langle\tilde{g}^{-1}\left(\left\{1+\left\{\sum_{j=1}^{n} \Theta_{j}\left(\frac{1-\tilde{g}_{\xi}^{j}}{\tilde{g}_{\xi}^{j}}\right)^{\phi}\right\}^{\frac{1}{\phi}}\right)^{-1}\right)\left(\mho^{(u)}\right), \tilde{g}^{-1}\left(1-\left(1+\left\{\sum_{j=1}^{n} \Theta_{j}\left(\frac{\tilde{g}_{\vartheta}^{j}}{1-\tilde{g}_{\vartheta}^{j}}\right)^{\phi}\right\}^{\frac{1}{\phi}}\right)^{-1}\right)\left(\mho^{(v)}\right)\right\rangle
\end{aligned}
$$

where $w_{j}(>0)$ is the weight of $\tilde{y}_{\wp}^{(j)}(\widetilde{\mho})$ such that $\sum_{j=1}^{n} w_{j}=1$, and $\Theta_{j}$ is given by Eq. (9).

Proof: Similar to Theorem 3.

Theorem 8: (Idempotency) Suppose $\tilde{y}_{\wp}^{(j)}(\widetilde{\delta})=<\left\{\wp_{\xi^{j(u)}}\left(\widetilde{J}^{(u)}\right)\right\},\left\{\wp_{g^{j(v)}}\left(\widetilde{\zeta}^{(v)}\right)\right\}>\left(j \in \Lambda_{n}\right)$ be a collection of

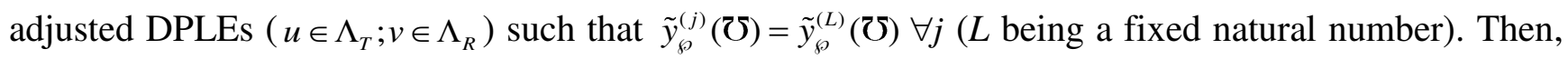

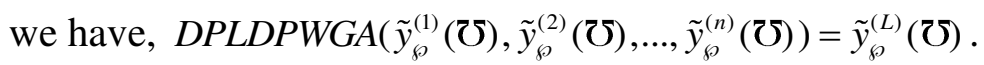

Proof: Similar to Theorem 4.

Theorem 9: (Monotonicity) Let $\tilde{y}_{\wp}^{(j)}(\widetilde{\mho})=<\left\{\wp_{\xi^{j(u)}}\left(\widetilde{J}^{(u)}\right)\right\},\left\{\wp_{g^{j(v)}}\left(\widetilde{J}^{(v)}\right)\right\}>$ and $\tilde{y}_{\wp}^{\prime(j)}(\widetilde{\mho})=<\left\{\wp_{\xi^{j(u)}}^{\prime}\left(\mho^{(u)}\right)\right\}$, $\left\{\wp_{y^{j(v)}}^{\prime}\left(\widetilde{\mho}^{(v)}\right)\right\}>\left(j \in \Lambda_{n}\right)$ be two collection of adjusted DPLEs $\left(u \in \Lambda_{T} ; v \in \Lambda_{R}\right)$ such that $\forall j, \wp_{\xi^{j(u)}} \leq \wp_{\xi^{j(u)}}^{\prime}$ and $\quad s_{g^{j(v)}} \geq s_{\vartheta^{j(v)}}^{\prime}$. Then, $\quad \operatorname{DPLDPWGA}\left(\tilde{y}_{\wp}^{(1)}(\widetilde{\mho}), \tilde{y}_{\wp}^{(2)}(\widetilde{\mho}), \ldots, \tilde{y}_{\wp}^{(n)}(\widetilde{\mho})\right) \leq D P L D P W G A$ $\left(\tilde{y}_{\wp}^{\prime(1)}(\widetilde{J}), \tilde{y}_{\wp}^{\prime(2)}(\widetilde{\zeta}), \ldots, \tilde{y}_{\wp}^{\prime(n)}(\widetilde{\mho})\right)$.

Proof: Similar to Theorem 5.

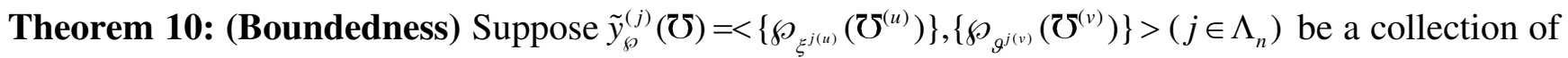
adjusted DPLEs $\quad\left(u \in \Lambda_{T} ; v \in \Lambda_{R} \quad\right)$.If $\quad \tilde{y}_{\wp}^{(j-)}(\widetilde{\zeta})=<\left\{\min _{u=1}^{T} \wp_{\xi^{j(u)}}\left(\boldsymbol{J}^{(u)}\right)\right\},\left\{\max _{v=1}^{R} \wp_{g^{j(v)}}\left(\widetilde{\zeta}^{(v)}\right)\right\}>$ and $\tilde{y}_{\wp}^{(j+)}(\widetilde{\mho})=<\left\{\max _{u=1}^{T} \wp_{\xi^{j(u)}}\left(\boldsymbol{\mho}^{(u)}\right)\right\},\left\{\min _{v=1}^{R} \wp_{g^{j(v)}}\left(\boldsymbol{J}^{(v)}\right)\right\}>, \quad$ then $\quad \tilde{y}_{\wp}^{(j-)}(\widetilde{\mho}) \prec \operatorname{DPLDPWGA}\left(\tilde{y}_{\wp}^{(1)}(\widetilde{\mho}), \tilde{y}_{\wp}^{(2)}(\widetilde{\mho}), \ldots\right.$, $\left.\tilde{y}_{\wp}^{(n)}(\widetilde{J})\right) \prec \tilde{y}_{\wp}^{(j+)}(\widetilde{\Xi)}$.

Proof: Similar to theorem 6.

\section{DPL FUCOM-MARCOS methodology for group decision-making:}

The procedural steps for FUCOM-MARCOS method under DPL environment are described below:

Step -1: To solve a MCGDM problem comprising $m$ different alternatives $A_{i}\left(i \in \Lambda_{m}\right)$ in which the alternatives are assessed by the decision experts $D_{k}\left(k \in \Lambda_{l}\right)$ in DPL environment under the set of $n$ distinct evaluation criteria $C_{j}\left(j \in \Lambda_{n}\right)$, we construct DPL-matrices representing the initial assessments of DEs.

Suppose $\quad \mathfrak{R}_{k}=\left[y_{\wp}^{(i j k)}(\boldsymbol{\mho})\right]_{m \times n}=\left[<\left\{\wp_{\xi^{i j k(u)}}\left(\boldsymbol{\mho}^{(u)}\right)\right\},\left\{\wp_{g^{j k(v)}}\left(\boldsymbol{\mho}^{(v)}\right)\right\}>\right]_{m \times n}\left(i \in \Lambda_{m}, j \in \Lambda_{n}, k \in \Lambda_{l}\right) \quad$ represents the initial assessment of the DE $D_{k}$. For assessment, we consider the LST $\Gamma=\left\{\wp_{\alpha}: \alpha=-\tau, \ldots ., 0, \ldots ., \tau\right\}$. Step-2: Obtain the initial assessment results of the DEs in terms of the adjusted DPLEs $\tilde{y}_{\wp}^{(i j k)}(\mho)\left(i \in \Lambda_{m}, j \in \Lambda_{n}, k \in \Lambda_{l}\right)$.

Step-3: Compute the supports $\operatorname{Supp}\left(\tilde{y}_{\wp}^{(i j k)}(\widetilde{\mho}), \tilde{y}_{\wp}^{(i j t)}(\widetilde{\mho})\right)\left(k, t \in \Lambda_{l} ; k \neq t\right)$ and is shown as 


$$
\operatorname{Supp}\left(\tilde{y}_{\wp}^{(i j k)}(\widetilde{Z}), \tilde{y}_{\wp}^{(i j t)}(\widetilde{J})\right)=1-\tilde{D}\left(\tilde{y}_{\wp}^{(i j k)}(\widetilde{Z}), \tilde{y}_{\wp}^{(i j t)}(\widetilde{J})\right) \quad\left(k, t \in \Lambda_{l} ; k \neq t\right)
$$

where $\tilde{D}\left(\tilde{y}_{\wp}^{(i j k)}(\widetilde{\delta}), \tilde{y}_{\wp}^{(i j t)}(\widetilde{\zeta})\right)$ is the distance between DPLEs $\tilde{y}_{\wp}^{(i j)}(\widetilde{\zeta})$ and $\tilde{y}_{\wp}^{(i j t)}(\widetilde{\zeta)}$. We define it by:

$$
\begin{aligned}
& \tilde{D}\left(\tilde{y}_{\wp}^{(i j k)}(\widetilde{\mho}), \tilde{y}_{\wp}^{(i j t)}(\boldsymbol{\mho})\right) \\
& =\sqrt{\frac{1}{2}\left(\sum_{u=1}^{\# T_{\xi}}\left(\sigma^{(u)} \times\left|\tilde{g}\left(\wp_{\xi^{j i k(u)}}\right)-\tilde{g}\left(\wp_{\xi^{j i t}(u)}\right)\right|\right)+\sum_{v=1}^{\# T_{g}}\left(\mho^{(v)} \times\left|\tilde{g}\left(\wp_{g^{j i k(v)}}\right)-\tilde{g}\left(\wp_{g^{j i t}(v)}\right)\right|\right)\right)}
\end{aligned}
$$

Step-4: Calculate the values $\Theta_{i j k}$ utilizing Eq. (15).

$$
\Theta_{i j k}=\frac{\varpi_{k}\left(1+\sum_{t=1, k \neq t}^{l} \operatorname{Supp}\left(\tilde{y}_{\wp}^{(i j k)}(\boldsymbol{\mho}), \tilde{y}_{\wp}^{(i j t)}(\boldsymbol{\mho})\right)\right)}{\sum_{k=1}^{l} \varpi_{k}\left(1+\sum_{t=1, k \neq t}^{l} \operatorname{Supp}\left(\tilde{y}_{\wp}^{(i j k)}(\boldsymbol{\delta}), \tilde{y}_{\wp}^{(i j t)}(\boldsymbol{\delta})\right)\right)}\left(i \in \Lambda_{m} ; j \in \Lambda_{n} ; k \in \Lambda_{l}\right)
$$

Here $\varpi_{k}\left(k \in \Lambda_{l}\right)$ are weights of the DEs $D_{k}\left(k \in \Lambda_{l}\right)$.

Clearly, $\sum_{k=1}^{l} \Theta_{i j k}=1$.

Step-5: Obtain the aggregated DPL matrix.

We use the DPLDPWAA operator or DPLDPWGA operator to get the aggregated DPL matrix $\left[\tilde{y}_{\wp}^{(i j)}(\boldsymbol{\sigma})\right]_{m \times n}$ as follows:

$\tilde{y}_{\wp}^{(i j)}(\boldsymbol{Z})$

$=\operatorname{DPLDPWAA}\left(\tilde{y}_{\wp}^{(i j))}(\widetilde{\mho}), \tilde{y}_{\wp}^{(i j 2)}(\widetilde{\mho}), \ldots \ldots . ., \tilde{y}_{\wp}^{(i j l)}(\widetilde{\mho})\right)$

$=\left\langle\tilde{g}^{-1}\left(1-\left(1+\left\{\sum_{k=1}^{l} \Theta_{i j k}\left(\frac{\tilde{g}_{\xi}^{i j k}}{1-\tilde{g}_{\xi}^{i j k}}\right)^{\phi}\right\}^{\frac{1}{\phi}}\right)\left(\mho^{(u)}\right), \tilde{g}^{-1}\left(\left(1+\left\{\sum_{k=1}^{l} \Theta_{i j k}\left(\frac{1-\tilde{g}_{\vartheta}^{i j k}}{\tilde{g}_{\vartheta}^{i j k}}\right)^{\phi}\right\}^{\frac{1}{\phi}}\right)^{-1}\right)\left(\mho^{(v)}\right)\right\rangle\right.$

or

$\tilde{y}_{\wp}^{(i j)}(\boldsymbol{Z})$

$=\operatorname{DPLDPWGA}\left(\tilde{y}_{\wp}^{(i j 1)}(\widetilde{\mho}), \tilde{y}_{\wp}^{(i j 2)}(\widetilde{\mho}), \ldots \ldots . ., \tilde{y}_{\wp}^{(i j l)}(\widetilde{\mho})\right)$

$$
=\left\langle\tilde{g}^{-1}\left(\left(1+\left\{\sum_{k=1}^{l} \Theta_{i j k}\left(\frac{1-\tilde{g}_{\xi}^{i j k}}{\tilde{g}_{\xi}^{i j k}}\right)^{\phi}\right\}^{\frac{1}{\phi}}\right)^{-1}\right)\left(\mho^{(u)}\right), \tilde{g}^{-1}\left(1-\left(1+\left\{\sum_{k=1}^{l} \Theta_{i j k}\left(\frac{\tilde{g}_{g}^{i j k}}{1-\tilde{g}_{\vartheta}^{i j k}}\right)^{\phi}\right\}^{\frac{1}{\phi}}\right)^{-1}\right)\left(\widetilde{\mho}^{(v)}\right)\right\rangle
$$

Step-6: Compute criteria weights using FUCOM.

Within the framework of MCDM, the calculation of the relative weights of criteria is considered as a tangible problem in MCDM, which is on the verge of subjectivity in some situations. Because of the crucial impact of weight coefficients on the solution in some methods, this procedure has been gaining in great importance and plays the major role in the final outcome of DM situations. The FUCOM method is used to compute the criteria weights in this study. According to the doctrines 
of comparisons in pairs of attributes and the validation of the outcomes by describing deviation from the utmost consistency, the full consistency method (FUCOM) is the best (Pamucar et al. 2018a). Badi and Abdulshahed (2019) used the FUCOM to evaluate airline traffic. Pamucar et al. (2018b) applied the FUCOM-MAIRCA procedure in order to evaluate level crossings in the security tool installation period. Noureddine and Ristic (2019) applied the FUCOM-MABAC approach so as to make an assessment of routes in hazardous product transport by road traffic. Fazlollahtabar et al. (2019) used FUCOM to select the equipment for storage schemes in logistics. Pamučar et al. (2019) proposed the fuzzy FUCOM-D'Bonferroni mean approach to the evaluation of Istanbul's urban mobility structure. For the purpose of establishing logistics firms, Yazdani et al. (2020) developed a two-phase DEAFUCOM-CoCoSo procedure under rough sets for the selection of a suitable region in the autonomous societies of Spain.

The steps showing how criteria weights are determined by applying FUCOM are presented below.

Step-6.1: At the very beginning, an attempt is made in this step towards ranking the assessment attributes $C_{1}, C_{2}, C_{3}, \ldots, C_{n}$. The priority order is defined based on the importance of the attributes, that is from the highest criteria significance to the lowest criteria significance. Thus, the obtained desired values of the weights enable us to frame the ranking of the criteria, which is as follows:

$$
C_{j(1)}>C_{j(2)}>C_{j(3)}>\ldots>C_{j(\sigma)}
$$

where $\sigma$ expresses the rank of the observed criterion. The sign "greater than" ( $>$ ) in Eq. (18) can be replaced with the sign "equal to" ("=") between these criteria while allowing the inference regarding the existence of at least two criteria with identical significance.

Step-6.2: This step is aimed at performing a comparative study of the ranked criteria and determining the comparative priority of the evaluation criteria as well $\eta_{\sigma /(\sigma+1)} ; \sigma \in \Lambda_{m}$. Importantly, the preference is given to the comparative priority $\eta_{\sigma /(\sigma+1)}$ of the evaluation criteria related to the rank $C_{j(\sigma)}$ while compared with that of the $C_{j(\sigma+1)}$. In this way, the expression responsible for the vectors of the comparative priorities associated with the corresponding assessment criteria can be suggested as follows:

$$
\psi=\eta_{1 / 2}, \eta_{2 / 3}, \ldots \ldots \ldots, \eta_{\sigma /(\sigma+1)}
$$

where significance is pursued by $\eta_{\sigma /(\sigma+1)}$, the criterion of the rank $C_{j(\sigma)}$ being assessed by the criterion of the rank $C_{j(\sigma+1)}$.

Step-6.3: This step requires that the outcomes of the weights of the considered criteria $\left(w_{1}, w_{2}, \ldots \ldots, w_{m}\right)^{\mathrm{T}}$ should be computed. The two constraints obeyed by the final results of the criteria weights are given below.

(I)The comparative priority among the considered criteria coincides with the ratio of the weight coefficients, i.e. the condition mentioned below must be satisfied:

$$
\frac{w_{\sigma}}{w_{\sigma+1}}=\eta_{\sigma /(\sigma+1)}
$$

(II) In addition to Eq. (20), the condition $\eta_{\sigma /(\sigma+1)} \times \eta_{(\sigma+1) /(\sigma+2)}=\eta_{\sigma /(\sigma+2)}$ must be met by the final values of the weights. Since $\eta_{\sigma /(\sigma+1)}=\frac{w_{\sigma}}{w_{\sigma+1}}$ and $\eta_{(\sigma+1) /(\sigma+2)}=\frac{w_{\sigma+1}}{w_{\sigma+2}}$, so $\frac{w_{\sigma}}{w_{\sigma+2}}=\frac{w_{\sigma}}{w_{\sigma+1}} \times \frac{w_{\sigma+1}}{w_{\sigma+2}}$ is obtained; therefore, there is the need to define an additional constraint for defining the final values of the weights of the considered attributes. That constraint is defined as follows: 


$$
\frac{w_{\sigma}}{w_{\sigma+2}}=\eta_{\sigma /(\sigma+1)} \times \eta_{(\sigma+1) /(\sigma+2)}
$$

Here, it is important to mention that the minimum deviation from the full consistency DFC $(\Omega)$ is only fulfilled if there is successful transitivity, i.e. only when both conditions $\frac{w_{\sigma}}{w_{\sigma+1}}=\eta_{\sigma /(\sigma+1)}$ and $\frac{w_{\sigma}}{w_{\sigma+2}}=\eta_{\sigma /(\sigma+1)} \times \eta_{(\sigma+1) /(\sigma+2)}$ are taken into consideration. For it to be implemented, the values of the weights $\quad\left(w_{1}, w_{2}, \ldots \ldots, w_{m}\right)^{\mathrm{T}}$ should comply with the conditions $\quad\left|\frac{w_{\sigma}}{w_{\sigma+1}}-\eta_{\sigma /(\sigma+1)}\right| \leq \Omega \quad$ and $\left|\frac{w_{\sigma}}{w_{\sigma+2}}-\eta_{\sigma /(\sigma+1)} \times \eta_{(\sigma+1) /(\sigma+2)}\right| \leq \Omega$, with the minimization of the value $\Omega$.

According to the constraints, the desired procedure for the calculation of the final values of the weights of the considered criteria is addressed as follows:

$$
\left.\begin{array}{l}
\operatorname{Min} \Omega \\
\left|\frac{w_{\sigma}}{w_{\sigma+1}}-\eta_{\sigma /(\sigma+1)}\right| \leq \Omega, \quad \forall \sigma \\
\left|\frac{w_{\sigma}}{w_{\sigma+2}}-\eta_{\sigma /(\sigma+1)} \times \eta_{(\sigma+1) /(\sigma+2)}\right| \leq \Omega, \quad \forall \sigma \\
w_{j} \geq 0, \forall j \\
\sum_{j=1}^{n} w_{j}=1
\end{array}\right\}
$$

By solving (22), the final weight values $\left(w_{1}, w_{2}, \ldots \ldots, w_{n}\right)^{\mathrm{T}}$ of the evaluation criteria are obtained.

Step-7: Form the extended aggregated (EA) decision matrix $\Re^{*}$.

In this step, the extension of the initial matrix is performed by introducing the ideal (ID) and antiideal (AID) solution. The AID solution is the worst alternative while the ID solution is an alternative with the best characteristic. Depending on profit and cost criteria, AID and ID are defined respectively as follows:

$$
\begin{aligned}
& y_{\mathfrak{\xi}}^{(j)(-)}(\mho)= \begin{cases}<\left\{\min _{i} \min _{u} \wp_{\xi^{j(u)}}\left(\min _{i, u} \mho^{(u)}\right)\right\},\left\{\max _{i} \max _{u} \wp_{g^{j(v)}}\left(\max _{i, v} \mho^{(v)}\right)\right\}>, & \text { if } C_{j} \in Q_{B} \\
\left\langle\left\{\max _{i} \max _{u} \wp_{g^{j(v)}}\left(\max _{i, u} \mho^{(v)}\right)\right\},\left\{\min _{i} \min _{u} \wp_{\xi^{j(u)}}\left(\min _{i, v} \mho^{(u)}\right)\right\}>,\right. & \text { if } C_{j} \in Q_{C}\end{cases} \\
& y_{\xi}^{(j)(+)}(\mho)= \begin{cases}<\left\{\max _{i} \max _{u} \wp_{\xi^{j(u)}}\left(\max _{i, u} \mho^{(u)}\right)\right\},\left\{\min _{i} \min _{u} \wp_{g^{j(v)}}\left(\min _{i, v} \mho^{(v)}\right)\right\}>, & \text { if } C_{j} \in Q_{B} \\
\left\langle\left\{\min _{i} \min _{u} \wp_{g^{j(v)}}\left(\min _{i, u} \mho^{(v)}\right)\right\},\left\{\max _{i} \max _{u} \wp_{\xi^{j(u(u)}}\left(\max _{i, v} \mho^{(u)}\right)\right\}>,\right. & \text { if } C_{j} \in Q_{C}\end{cases}
\end{aligned}
$$

Here, $Q_{B}, Q_{C}$ refers the collection of all profit and cost criteria respectively.

The EA decision matrix $\Re^{*}$ is given by: 


\begin{tabular}{|c|c|c|c|c|}
\hline & $C_{1}$ & $C_{2}$ & $\ldots \ldots$ & $C_{n}$ \\
\hline$A I D$ & $y_{\wp}^{(1)(-)}(\boldsymbol{Z})$ & $y_{\wp}^{(2)(-)}(\mathbf{W})$ & $\ldots . .$. & $y_{\wp}^{(n)(-)}(\widetilde{Z})$ \\
\hline$A_{1}$ & $\tilde{y}_{\wp}^{(11)}(\boldsymbol{Z})$ & $\tilde{y}_{\wp}^{(12)}(\boldsymbol{Z})$ & $\ldots .$. & $\tilde{y}_{\wp}^{(1 n)}(\widetilde{J})$ \\
\hline$A_{2}$ & $\tilde{y}_{\wp}^{(21)}(\boldsymbol{J})$ & $\tilde{y}_{\wp}^{(22)}(\boldsymbol{\delta})$ & $\ldots$. & $\tilde{y}_{\wp}^{(2 n)}(\boldsymbol{Z})$ \\
\hline$\vdots$ & $\ldots .$. & $\ldots \ldots$ & $\ldots$. & $\ldots .$. \\
\hline$A_{m}$ & $\tilde{y}_{\wp}^{(m 1)}(\boldsymbol{W})$ & $\tilde{y}_{\wp}^{(m 2)}(\mathbf{W})$ & $\ldots .$. & $\tilde{y}_{\wp}^{(m n)}(\boldsymbol{Z})$ \\
\hline$I D$ & $y_{\wp}^{(1)(+)}(\mathbf{Z})$ & $y_{\wp}^{(2)(+)}(\boldsymbol{J})$ & $\ldots \ldots$ & $y_{\wp}^{(n)(+)}(\mathbf{Z})$ \\
\hline
\end{tabular}

Step-8: Obtain the normalized EA decision matrix $\mathfrak{R}^{* N}=\left[\hat{y}_{\wp}^{(i j)}(\boldsymbol{\mho})\right]_{m \times n}=\left[<\left\{\hat{\wp}_{\xi^{i j(u)}}\left(\boldsymbol{J}^{(u)}\right)\right\},\left\{\hat{\wp}_{g^{i j(v)}}\left(\boldsymbol{\mho}^{(v)}\right)\right\}>\right]_{m \times n}$.

The elements of the normalization matrix are obtained by applying Eq. (25).

$$
\hat{y}_{s}^{(i j)}(\boldsymbol{Z})=\left\{\begin{array}{ll}
\left\langle\left\{\wp_{\tilde{g}_{\xi}^{i j} / g_{\xi}^{j(+)}}\left(\boldsymbol{J}^{(u)}\right)\right\},\left\{\wp_{\tilde{g}_{g}^{i j} / g_{g}^{j(+)}}\left(\boldsymbol{J}^{(v)}\right)\right\}\right\rangle, & \text { if } C_{j} \in Q_{B} \\
\left\langle\left\{\wp_{\tilde{g}_{\xi}^{j(+)} / \tilde{g}_{\xi}^{i j}}\left(\boldsymbol{J}^{(u)}\right)\right\},\left\{\wp_{\tilde{g}_{g}^{j(+)} / \tilde{g}_{9}^{i j}}\left(\boldsymbol{J}^{(v)}\right)\right\}\right\rangle, & \text { if } C_{j} \in Q_{C}
\end{array} \quad\left(i \in \Lambda_{m}, j \in \Lambda_{n}\right)\right.
$$

Step-9: Construct the weighted normalized EA decision matrix $\mathfrak{R}^{* N W}=\left[\hat{y}_{\wp}^{(i j)}(\boldsymbol{Z})\right]_{m \times n}$.

The elements of this matrix are defined as:

$\left.\hat{y}_{\wp}^{(i j)}(\boldsymbol{\mho})=<\wp_{w_{j} \times \tilde{g}_{\xi}^{i j(u)}}\left(\boldsymbol{\mho}^{(u)}\right)\right\},\left\{\wp_{w_{j} \times \tilde{g}_{g}^{i j(v)}}\left(\boldsymbol{\mho}^{(v)}\right)>\left(i \in \Lambda_{m}, j \in \Lambda_{n}\right)\right.$.

Suppose, $\hat{y}_{\wp}^{\prime(i j)}(\boldsymbol{\mho})=<\left\{\hat{\wp}_{\xi^{i j(u)}}^{\prime}\left(\boldsymbol{\mho}^{(u)}\right)\right\},\left\{\hat{\wp}_{g^{i j(v)}}^{\prime}\left(\boldsymbol{\mho}^{(v)}\right)\right\}>\left(i \in \Lambda_{m}, j \in \Lambda_{n}\right)$

Step-10: Find the utility degree of $A_{i}\left(i \in \Lambda_{m}\right)$.

The utility degree of an alternative in relation to AID and ID solution are calculated based on Eq. (26) and Eq. (27).

$$
\begin{aligned}
& \hbar_{i}^{-}=\frac{\sum_{j=1}^{n} S\left(\hat{y}_{\wp}^{\prime(i j)}(\boldsymbol{\mho})\right)}{\sum_{j=1}^{n} S\left(\hat{y}_{\wp}^{\prime(j)(-)}(\boldsymbol{\mho})\right)} \quad\left(i \in \Lambda_{m}\right) \\
& \hbar_{i}^{+}=\frac{\sum_{j=1}^{n} S\left(\hat{y}_{\wp}^{(i j)}(\boldsymbol{\mho})\right)}{\sum_{j=1}^{n} S\left(\hat{y}_{\wp}^{(j)(+)}(\widetilde{\mho})\right)} \quad\left(i \in \Lambda_{m}\right)
\end{aligned}
$$

Step 11: Determine the utility values connected with the AID and ID solutions.

The utility values for the AID and ID solutions are given respectively by: 


$$
\begin{aligned}
& {\lambda_{i}^{-}}^{=}=\frac{\hbar_{i}^{+}}{\hbar_{i}^{-}+\hbar_{i}^{+}}\left(i \in \Lambda_{m}\right) \\
& \lambda_{i}^{+}=\frac{\hbar_{i}^{-}}{\hbar_{i}^{-}+\hbar_{i}^{+}}\left(i \in \Lambda_{m}\right)
\end{aligned}
$$

Step 12: Determine the overall utility value of $A_{i}\left(i \in \Lambda_{m}\right)$.

The overall utility value of each alternative is calculated based on Eq. (30).

$$
\lambda_{i}=\frac{\hbar_{i}^{+}+\hbar_{i}^{-}}{1+\frac{1-\lambda_{i}^{+}}{\lambda_{i}^{+}}+\frac{1-\lambda_{i}^{-}}{\lambda_{i}^{-}}} \quad\left(i \in \Lambda_{m}\right)
$$

Step 13: Rank the efficient alternatives $A_{i}\left(i \in \Lambda_{m}\right)$ and choose the best one.

Ranking of alternatives is based on their overall utility values. It is desirable that the optimal alternative should have the maximum possible utility value.

\section{Case study:}

\subsection{Problem description:}

These days, because of the progressions in data innovations, educational institutions, research centers, and even the people have agreed on the utilization of the Internet in their everyday exercises. Learning methods have likewise been influenced by the energetic development in Information and Communication Technology (ICT) and the Internet region, which achieve the arrangement of novel learning settings. The advanced and reasonable technologies enlivened many educational establishments to have an alternate of options in contrast to ordinary classroom instruction. Additionally, the progressions in the Internet innovations cover the mode for the establishment of a novel instructive framework called electronic learning (e-learning), where education and knowledge are being conveyed to students using the Internet or associated web platforms with a decent norm and with no impediment to a specific area. It includes utilizing multimedia that incorporates audio, video, animations, and text illustrations. The most general type of e-learning with the software application is termed as a learning management system (LMS) Also, the execution of LMS wants vast sums of money and assurance from the institutes and organizations.

An LMS is characterized as a software procedure which oversees, record, track, reporting as well as the conveyance of education materials and training, which gives a way to handily follow and try out an appropriate learning action to attain advance skills. In other words, an LMS can be portrayed as a process that involves diverse procedures that assist learning and teaching actions. The use of LMS these days becomes a fundamental column that underpins and advances educational platforms, the fast development of computer and Internet-based tools creates a generous amount of LMSs accessible over the Internet, both the authorized and the free versions. During these years, educational organizations capitalized on massive amounts of money and spend momentous amounts of time executing LMSs. Various LMS have been presented as OSS licenses such as Canvas, Moodle, Sakai, ATutor, Dokeos, eFront, etc. These LMS are exceptionally valuable for e-learning. OSS is a tool-free of license expenses and distributed with its computer program source code. It is designated as a process to address the increasing costs of campus-wide software applications while empowering the formation of learner-centered structures. The assessment of unseemly OSS learning management system (OSSLMS) package unfavorably affects the business procedures and tasks of an institution. The assignment of OSS-LMS determination has become progressively unpredictable due to the troubles in the choice 
of suitable OSS-LMS for business requirements specified a huge amount of OSS-LMS available on the market, the deficiency of experience and practical knowledge of the DMs, and the continuous advancement in the discipline of information technology. This issue can be solved by utilizing MCDM techniques as it includes multiple attributes to be inspected and investigated before making assessments. Thus, the appraisal of OSS-LMS packages is a complicated MCDM that can be estimated over various clashing criteria.

Suppose, an educational institute wants to select an efficient OSS-LMS package out of three OSSLMS packages, namely- Moodle $\left(\mathrm{A}_{1}\right)$, ATutor $\left(\mathrm{A}_{2}\right)$, and eFront $\left(\mathrm{A}_{3}\right)$. These three OSS-LMS are considered as alternatives in this study that are to be assessed based on four evaluation criteria, namely- Functionality $\left(\mathrm{C}_{1}\right)$, E-learning standards $\left(\mathrm{C}_{2}\right)$, Reliability $\left(\mathrm{C}_{3}\right)$, and Learner's communication $\left(\mathrm{C}_{4}\right)$. The DEs utilize the assessment procedure of three OSS-LMS subject to available DPL information under the aforesaid four criteria.

\subsection{Solution}

To solve the OSS-LMS selection problem described above, we utilize the developed FUCOMMARCOS method for the evaluation of alternatives with DPL information. The proposed framework encompasses the consecutive steps as:

Step-1: In this step, the decision experts will assess the options $A_{i}\left(i \in \Lambda_{3}\right)$ in relation to considered attributes $C_{j}\left(j \in \Lambda_{4}\right)$. DEs give their assessment results with the LST $\Gamma=\left\{\wp_{\alpha}: \alpha=-3,-2,-1,0,1,2,3\right\}$ where $\wp_{-3}=$ extremely poor, $\wp_{-2}=$ poor, $\wp_{-1}=$ moderately poor, $\wp_{0}=$ fair, $\wp_{1}=$ good, $\wp_{2}=$ very good, and $\wp_{3}=$ excellent. The initial assessment results are given in the form of the matrices $\mathfrak{R}_{k}=\left[\tilde{y}_{\wp}^{(i j k)}(\boldsymbol{\mho})\right]_{3 \times 4}=\left[<\left\{\wp_{\xi^{j i k(u)}}\left(\boldsymbol{J}^{(u)}\right)\right\},\left\{\wp_{\xi^{j k(v)}}\left(\boldsymbol{J}^{(v)}\right)\right\}>\right]_{3 \times 4}\left(i \in \Lambda_{3}, j \in \Lambda_{4}, k \in \Lambda_{3}\right)$ is given in the form of Table- 2 .

Table-2: Initial decision matrix

\begin{tabular}{|c|c|c|c|c|c|}
\hline & & $\mathrm{C}_{1}$ & $\mathrm{C}_{2}$ & $\mathrm{C}_{3}$ & $\mathrm{C}_{4}$ \\
\hline \multirow{3}{*}{$\mathrm{D}_{1}$} & $\mathrm{~A}_{1}$ & $\begin{array}{c}<\left\{\wp_{-2}(0.3), \wp_{-1}(0.4),\right. \\
\left.\wp_{1}(0.3)\right\},\left\{\wp_{-1}(0.3),\right. \\
\left.\wp_{0}(0.7)\right\}>\end{array}$ & $\begin{array}{l}<\left\{\wp_{1}(0.6), \wp_{2}(0.4)\right\}, \\
\left\{\wp_{-2}(0.6), \wp_{1}(0.4)\right\}>\end{array}$ & $\begin{array}{c}<\left\{\wp_{-1}(0.6), \wp_{1}(0.4)\right\}, \\
\left\{\wp_{0}(0.1), \wp_{2}(0.9)\right\}>\end{array}$ & $\begin{array}{c}<\left\{\wp_{0}(1)\right\}, \\
\left\{\wp_{-2}(0.3), \wp_{-1}(0.6),\right. \\
\left.\wp_{1}(0.1)\right\}>\end{array}$ \\
\hline & $\mathrm{A}_{2}$ & $\begin{array}{c}<\left\{\wp_{-1}(0.1), \wp_{0}(0.9)\right\}, \\
\left\{\wp_{-2}(0.6), \wp_{-1}(0.3),\right. \\
\left.\wp_{1}(0.1)\right\}>\end{array}$ & $\begin{array}{l}<\left\{\wp_{-2}(0.7), \wp_{1}(0.3)\right\}, \\
\left\{\wp_{1}(0.3), \wp_{4}(0.6)\right\}>\end{array}$ & $\begin{array}{l}<\left\{\wp_{0}(0.2), \wp_{2}(0.8)\right\}, \\
\left\{\wp_{-1}(0.8), \wp_{1}(0.2)\right\}>\end{array}$ & $\begin{array}{c}<\left\{\wp_{-2}(0.2), \wp_{-1}(0.3),\right. \\
\left.\wp_{1}(0.5)\right\},\left\{\wp_{0}(1)\right\}>\end{array}$ \\
\hline & $\mathrm{A}_{3}$ & $\begin{array}{c}<\left\{\wp_{1}(1)\right\}, \\
\left\{\wp_{0}(0.1), \wp_{2}(0.9)\right\}>\end{array}$ & $\begin{array}{c}<\left\{\wp_{-1}(0.3), \wp_{0}(0.7)\right\}, \\
\left\{\wp_{-2}(0.5), \wp_{-1}(0.3),\right. \\
\left.\wp_{1}(0.2)\right\}>\end{array}$ & $\begin{array}{l}<\left\{\wp_{-2}(0.8), \wp_{-1}(0.2)\right\}, \\
\left\{\wp_{-1}(0.2), \wp_{0}(0.8)\right\}>\end{array}$ & $\begin{array}{c}<\left\{\wp_{2}(1)\right\}, \\
\left\{\wp_{-2}(0.3), \wp_{1}(0.7)\right\}>\end{array}$ \\
\hline \multirow{4}{*}{$\mathrm{D}_{2}$} & $\mathrm{~A}_{1}$ & $\begin{array}{l}<\left\{\wp_{-1}(0.7), \wp_{1}(0.3)\right\}, \\
\left\{\wp_{-2}(0.7), \wp_{-1}(0.3)\right\}>\end{array}$ & $\begin{array}{c}<\left\{\wp_{-2}(0.6), \wp_{-1}(0.4)\right\}, \\
\left\{\wp_{2}(1)\right\}>\end{array}$ & $\begin{array}{c}<\left\{\wp_{-2}(0.1), \wp_{-1}(0.5),\right. \\
\left.\wp_{1}(0.4)\right\},\left\{\wp_{1}(1)\right\}>\end{array}$ & $\begin{array}{c}<\left\{\wp_{1}(0.5), \wp_{2}(0.5)\right\}, \\
\left\{\wp_{-1}(1)\right\}>\end{array}$ \\
\hline & $\mathrm{A}_{2}$ & $\begin{array}{l}<\left\{\wp_{0}(0.1), \wp_{2}(0.9)\right\}, \\
\left\{\wp_{-1}(0.9), \wp_{1}(0.1)\right\}>\end{array}$ & $\begin{aligned} &<\left\{\wp_{-2}(0.5), \wp_{-1}(0.3),\right. \\
&\left.\wp_{1}(0.2)\right\},\left\{\wp_{0}(1)\right\}>\end{aligned}$ & $\begin{array}{c}<\left\{\wp_{-1}(0.2), \wp_{0}(0.8)\right\}, \\
\left\{\wp_{-2}(0.1), \wp_{-1}(0.7),\right. \\
\left.\wp_{1}(0.2)\right\}>\end{array}$ & $\begin{array}{l}<\left\{\wp_{-2}(0.3), \wp_{1}(0.7)\right\}, \\
\left\{\wp_{1}(0.3), \wp_{2}(0.7)\right\}>\end{array}$ \\
\hline & $\mathrm{A}_{3}$ & $\begin{array}{l}\left\langle\left\{\wp_{-2}(0.9), \wp_{-1}(0.1)\right\},\right. \\
\left\{\wp_{-1}(0.8), \wp_{1}(0.2)\right\}>\end{array}$ & $\begin{array}{c}<\left\{\wp_{2}(1)\right\}, \\
\left\{\wp_{-2}(0.7), \wp_{-1}(0.3)\right\}>\end{array}$ & $\begin{array}{l}<\left\{\wp_{1}(1)\right\},\left\{\wp_{-2}(0.2),\right. \\
\left.\wp_{-1}(0.7), \wp_{1}(0.1)\right\}>\end{array}$ & $\begin{array}{l}<\left\{\wp_{-1}(0.3), \wp_{0}(0.7)\right\}, \\
\left\{\wp_{1}(0.3), \wp_{0}(0.7)\right\}>\end{array}$ \\
\hline & $\overline{A_{1}}$ & $<\left\{\wp_{0}(0.3), \wp_{2}(0.7)\right\}$ & $<\left\{\wp_{-2}(0.4), \wp_{-1}(0.5)\right.$, & $<\left\{\wp_{-1}(0.1), \wp_{1}(0.3)\right.$, & $<\left\{\wp_{-2}(0.5), \wp_{1}(0.5)\right\}$, \\
\hline
\end{tabular}




\begin{tabular}{|c|c|c|c|c|c|}
\hline \multirow{3}{*}{$\mathrm{D}_{3}$} & & $\left\{\wp_{-2}(0.4), \wp_{-1}(0.6)\right\}>$ & $\left.\wp_{1}(0.1)\right\},\left\{\wp_{2}(1)\right\}>$ & $\left.\wp_{0}(0.6)\right\},\left\{\wp_{1}(1)\right\}>$ & $\left\{\wp_{-1}(0.6), \wp_{0}(0.4)\right\}>$ \\
\hline & $\mathrm{A}_{2}$ & $\begin{array}{l}<\left\{\wp_{-2}(0.8), \wp_{-1}(0.2)\right\}, \\
\left\{\wp_{0}(0.6), \wp_{2}(0.4)\right\}>\end{array}$ & $\begin{array}{l}<\left\{\wp_{2}(1)\right\},\left\{\wp_{-2}(0.2),\right. \\
\left.\wp_{-1}(0.2), \wp_{1}(0.6)\right\}>\end{array}$ & $\begin{array}{c}<\left\{\wp_{1}(1)\right\}, \\
\left\{\wp_{-1}(0.1), \wp_{0}(0.9)\right\}>\end{array}$ & $\begin{array}{l}\left\langle\left\{\wp_{-1}(0.3), \wp_{0}(0.7)\right\},\right. \\
\left\{\wp_{-2}(0.3), \wp_{1}(0.7)\right\}>\end{array}$ \\
\hline & $\mathrm{A}_{3}$ & $\begin{array}{l}\left\langle\left\{\wp_{-1}(0.9), \wp_{1}(0.1)\right\},\right. \\
\left\{\wp_{-1}(0.8), \wp_{1}(0.2)\right\}>\end{array}$ & $\left\langle\left\{\wp_{0}(1)\right\},\left\{\wp_{-1}(1)\right\}>\right.$ & $\begin{array}{l}<\left\{\wp_{-2}(0.1), \wp_{-1}(0.7),\right. \\
\left.\wp_{1}(0.2)\right\},\left\{\wp_{-2}(0.2),\right. \\
\left.\wp_{-1}(0.7), \wp_{1}(0.1)\right\}>\end{array}$ & $\begin{array}{c}<\left\{\wp_{1}(0.3), \wp_{2}(0.7)\right\}, \\
\left\{\wp_{1}(1)\right\}>\end{array}$ \\
\hline
\end{tabular}

Step-2: The initial assessment results of the DEs in terms of adjusted DPLEs are given in Table-3.

Table-3: Adjusted Initial decision matrix

\begin{tabular}{|c|c|c|c|c|c|}
\hline & & $\mathrm{C}_{1}$ & $\mathrm{C}_{2}$ & $\mathrm{C}_{3}$ & $\mathrm{C}_{4}$ \\
\hline \multirow{3}{*}{$\mathrm{D}_{1}$} & $\mathrm{~A}_{1}$ & $\begin{array}{l}<\left\{\wp_{-2}(0.3), \wp_{-1}(0.2),\right. \\
\left.\wp_{-1}(0.2), \wp_{1}(0.3)\right\}, \\
\left\{\wp_{-1}(0.3), \wp_{0}(0.1),\right. \\
\left.\wp_{0}(0.3), \wp_{0}(0.3)\right\}>\end{array}$ & $\begin{array}{c}<\left\{\wp_{1}(0.4), \wp_{1}(0.2),\right. \\
\left.\wp_{2}(0.3), \wp_{2}(0.1)\right\}, \\
\left\{\wp_{-2}(0.3), \wp_{-2}(0.3),\right. \\
\left.\wp_{1}(0.1), \wp_{1}(0.3)\right\}>\end{array}$ & $\begin{array}{l}<\left\{\wp_{-1}(0.1), \wp_{-1}(0.3),\right. \\
\left.\wp_{-1}(0.2), \wp_{1}(0.4)\right\}, \\
\left\{\wp_{0}(0.1), \wp_{2}(0.3),\right. \\
\left.\wp_{2}(0.3), \wp_{2}(0.3)\right\}>\end{array}$ & $\begin{array}{l}<\left\{\wp_{0}(0.2), \wp_{0}(0.3),\right. \\
\left.\wp_{0}(0.3), \wp_{0}(0.2)\right\}, \\
\left\{\wp_{-2}(0.3), \wp_{-1}(0.3),\right. \\
\left.\wp_{-1}(0.3), \wp_{1}(0.1)\right\}>\end{array}$ \\
\hline & $\mathrm{A}_{2}$ & $\begin{array}{c}<\left\{\wp_{-1}(0.1), \wp_{0}(0.5),\right. \\
\left.\wp_{0}(0.2), \wp_{0}(0.2)\right\}, \\
\left\{\wp_{-2}(0.6), \wp_{-1}(0.1),\right. \\
\left.\wp_{-1}(0.2), \wp_{1}(0.1)\right\}>\end{array}$ & $\begin{array}{c}<\left\{\wp_{-2}(0.5), \wp_{-2}(0.2),\right. \\
\left.\wp_{1}(0.1), \wp_{1}(0.2)\right\}, \\
\left\{\wp_{1}(0.2), \wp_{1}(0.1),\right. \\
\left.\wp_{2}(0.1), \wp_{2}(0.6)\right\}>\end{array}$ & $\begin{array}{c}<\left\{\wp_{0}(0.2), \wp_{2}(0.2),\right. \\
\left.\wp_{2}(0.5), \wp_{2}(0.1)\right\}, \\
\left\{\wp_{-1}(0.1), \wp_{-1}(0.6),\right. \\
\left.\wp_{-1}(0.1), \wp_{1}(0.2)\right\}>\end{array}$ & $\begin{array}{c}<\left\{\wp_{-2}(0.2), \wp_{-1}(0.1),\right. \\
\left.\wp_{-1}(0.2), \wp_{1}(0.5)\right\}, \\
\left\{\wp_{0}(0.1), \wp_{0}(0.2),\right. \\
\left.\wp_{0}(0.6), \wp_{0}(0.1)\right\}>\end{array}$ \\
\hline & $\mathrm{A}_{3}$ & $\begin{array}{l}<\left\{\wp_{1}(0.6), \wp_{1}(0.1),\right. \\
\left.\wp_{1}(0.2), \wp_{1}(0.1)\right\}, \\
\left\{\wp_{0}(0.1), \wp_{2}(0.5),\right. \\
\left.\wp_{2}(0.2), \wp_{2}(0.2)\right\}>\end{array}$ & $\begin{array}{c}<\left\{\wp_{-1}(0.2), \wp_{-1}(0.1),\right. \\
\left.\wp_{0}(0.1), \wp_{0}(0.6)\right\}, \\
\left\{\wp_{-2}(0.5), \wp_{-1}(0.2),\right. \\
\left.\wp_{-1}(0.1), \wp_{1}(0.2)\right\}>\end{array}$ & $\begin{array}{l}<\left\{\wp_{-2}(0.1), \wp_{-2}(0.6),\right. \\
\left.\wp_{-2}(0.1), \wp_{-1}(0.2)\right\}, \\
\left\{\wp_{-1}(0.2), \wp_{0}(0.2),\right. \\
\left.\wp_{0}(0.5), \wp_{0}(0.1)\right\}>\end{array}$ & $\begin{array}{c}<\left\{\wp_{2}(0.1), \wp_{2}(0.2),\right. \\
\left.\wp_{2}(0.6), \wp_{2}(0.1)\right\}, \\
\left\{\wp_{-2}(0.2), \wp_{-2}(0.1),\right. \\
\left.\wp_{1}(0.2), \wp_{1}(0.5)\right\}>\end{array}$ \\
\hline \multirow{3}{*}{$\mathrm{D}_{2}$} & $\mathrm{~A}_{1}$ & $\begin{array}{c}\left\langle\left\{\wp_{-1}(0.3), \wp_{-1}(0.2),\right.\right. \\
\left.\wp_{-1}(0.2), \wp_{1}(0.3)\right\}, \\
\left\{\wp_{-2}(0.3), \wp_{-2}(0.1),\right. \\
\left.\wp_{-2}(0.3), \wp_{-1}(0.3)\right\}>\end{array}$ & $\begin{array}{c}<\left\{\wp_{-2}(0.4), \wp_{-2}(0.2),\right. \\
\left.\wp_{-1}(0.3), \wp_{-1}(0.1)\right\}, \\
\left\{\wp_{2}(0.3), \wp_{2}(0.3),\right. \\
\left.\wp_{2}(0.1), \wp_{2}(0.3)\right\}>\end{array}$ & $\begin{array}{c}<\left\{\wp_{-2}(0.1), \wp_{-1}(0.3),\right. \\
\left.\wp_{-1}(0.2), \wp_{1}(0.4)\right\}, \\
\left\{\wp_{1}(0.1), \wp_{1}(0.3),\right. \\
\left.\wp_{1}(0.3), \wp_{1}(0.3)\right\}>\end{array}$ & $\begin{array}{c}\left\{\wp_{1}(0.2), \wp_{1}(0.3),\right. \\
\left.\wp_{2}(0.3), \wp_{2}(0.2)\right\}, \\
\left\{\wp_{-1}(0.3), \wp_{-1}(0.3),\right. \\
\left.\wp_{-1}(0.3), \wp_{-1}(0.1)\right\}>\end{array}$ \\
\hline & $\mathrm{A}_{2}$ & $\begin{array}{c}<\left\{\wp_{0}(0.1), \wp_{2}(0.5),\right. \\
\left.\wp_{2}(0.2), \wp_{2}(0.2)\right\}, \\
\left\{\wp_{-1}(0.6), \wp_{-1}(0.1),\right. \\
\left.\wp_{-1}(0.2), \wp_{1}(0.1)\right\}>\end{array}$ & $\begin{array}{c}<\left\{\wp_{-2}(0.5), \wp_{-1}(0.2),\right. \\
\left.\wp_{-1}(0.1), \wp_{1}(0.2)\right\}, \\
\left\{\wp_{0}(0.2), \wp_{0}(0.1),\right. \\
\left.\wp_{0}(0.1), \wp_{0}(0.6)\right\}>\end{array}$ & $\begin{array}{c}<\left\{\wp_{-1}(0.2), \wp_{0}(0.2),\right. \\
\left.\wp_{0}(0.5), \wp_{0}(0.1)\right\}, \\
\left\{\wp_{-2}(0.1), \wp_{-1}(0.6),\right. \\
\left.\wp_{-1}(0.1), \wp_{1}(0.2)\right\}>\end{array}$ & $\begin{array}{c}<\left\{\wp_{-2}(0.2), \wp_{-2}(0.1),\right. \\
\left.\wp_{1}(0.2), \wp_{1}(0.5)\right\}, \\
\left\{\wp_{1}(0.1), \wp_{1}(0.2),\right. \\
\left.\wp_{2}(0.6), \wp_{2}(0.1)\right\}>\end{array}$ \\
\hline & $\mathrm{A}_{3}$ & $\begin{array}{l}<\left\{\wp_{-2}(0.6), \wp_{-2}(0.1),\right. \\
\left.\wp_{-2}(0.2), \wp_{-1}(0.1)\right\}, \\
\left\{\wp_{-1}(0.1), \wp_{-1}(0.5),\right. \\
\left.\wp_{-1}(0.2), \wp_{1}(0.2)\right\}>\end{array}$ & $\begin{array}{c}<\left\{\wp_{2}(0.2), \wp_{2}(0.1),\right. \\
\left.\wp_{2}(0.1), \wp_{2}(0.6)\right\}, \\
\left\{\wp_{-2}(0.5), \wp_{-2}(0.2),\right. \\
\left.\wp_{-1}(0.1), \wp_{-1}(0.2)\right\}>\end{array}$ & $\begin{array}{c}<\left\{\wp_{1}(0.1), \wp_{1}(0.6),\right. \\
\left.\wp_{1}(0.1), \wp_{1}(0.2)\right\}, \\
\left\{\wp_{-2}(0.2), \wp_{-1}(0.2),\right. \\
\left.\wp_{-1}(0.5), \wp_{1}(0.1)\right\}>\end{array}$ & $\begin{array}{c}<\left\{\wp_{-1}(0.1), \wp_{-1}(0.2),\right. \\
\left.\wp_{0}(0.6), \wp_{0}(0.1)\right\}, \\
\left\{\wp_{1}(0.2), \wp_{1}(0.1),\right. \\
\left.\wp_{0}(0.2), \wp_{0}(0.5)\right\}>\end{array}$ \\
\hline & $\mathrm{A}_{1}$ & $\begin{array}{c}<\left\{\wp_{0}(0.3), \wp_{2}(0.2),\right. \\
\left.\wp_{2}(0.2), \wp_{2}(0.3)\right\}, \\
\left\{\wp_{-2}(0.3), \wp_{-2}(0.1),\right. \\
\left.\wp_{-1}(0.3), \wp_{-1}(0.3)\right\}>\end{array}$ & $\begin{array}{c}<\left\{\wp_{-2}(0.4), \wp_{-1}(0.2),\right. \\
\left.\wp_{-1}(0.3), \wp_{1}(0.1)\right\}, \\
\left\{\wp_{2}(0.3), \wp_{2}(0.3),\right. \\
\left.\wp_{2}(0.1), \wp_{2}(0.3)\right\}>\end{array}$ & $\begin{array}{l}\left\langle\left\{\wp_{-1}(0.1), \wp_{1}(0.3),\right.\right. \\
\left.\wp_{0}(0.2), \wp_{0}(0.4)\right\}, \\
\left\{\wp_{1}(0.1), \wp_{1}(0.3),\right. \\
\left.\wp_{1}(0.3), \wp_{1}(0.3)\right\}>\end{array}$ & $\begin{array}{c}<\left\{\wp_{-2}(0.2), \wp_{-2}(0.3),\right. \\
\left.\wp_{1}(0.3), \wp_{1}(0.2)\right\}, \\
\left\{\wp_{-1}(0.3), \wp_{-1}(0.3),\right. \\
\left.\wp_{0}(0.3), \wp_{0}(0.1)\right\}>\end{array}$ \\
\hline & $\mathrm{A}_{2}$ & $\begin{array}{l}<\left\{\wp_{-2}(0.1), \wp_{-2}(0.5),\right. \\
\left.\wp_{-2}(0.2), \wp_{-1}(0.2)\right\}, \\
\left\{\wp_{0}(0.6), \wp_{2}(0.1),\right.\end{array}$ & $\begin{array}{l}<\left\{\wp_{2}(0.5), \wp_{2}(0.2),\right. \\
\left.\wp_{2}(0.1), \wp_{2}(0.2)\right\}, \\
\left\{\wp_{-2}(0.2), \wp_{-1}(0.1),\right.\end{array}$ & $\begin{array}{l}<\left\{\wp_{1}(0.2), \wp_{1}(0.2),\right. \\
\left.\wp_{1}(0.5), \wp_{1}(0.1)\right\}, \\
\left\{\wp_{-1}(0.1), \wp_{0}(0.6),\right.\end{array}$ & $\begin{array}{c}<\left\{\wp_{-1}(0.2), \wp_{-1}(0.1),\right. \\
\left.\wp_{0}(0.2), \wp_{0}(0.5)\right\}, \\
\left\{\wp_{-2}(0.1), \wp_{-2}(0.2),\right.\end{array}$ \\
\hline
\end{tabular}




\begin{tabular}{|c|c|c|c|c|c|}
\hline $\mathrm{D}_{3}$ & $\left.\left.\wp_{2}(0.2), \wp_{2}(0.1)\right\}\right\rangle$ & $\left.\left.\wp_{-1}(0.1), \wp_{1}(0.6)\right\}\right\rangle$ & $\left.\left.\wp_{0}(0.1), \wp_{0}(0.2)\right\}\right\rangle$ & $\left.\left.\wp_{1}(0.6), \wp_{1}(0.1)\right\}\right\rangle$ \\
\cline { 2 - 7 } & $\mathrm{A}_{3}$ & $\left\langle\left\{\wp_{-1}(0.6), \wp_{-1}(0.1)\right.\right.$, & $\left\langle\left\{\wp_{0}(0.2), \wp_{0}(0.1)\right.\right.$, & $\left\langle\left\{\wp_{-2}(0.1), \wp_{-1}(0.6)\right.\right.$, & $\left\langle\left\{\wp_{1}(0.1), \wp_{1}(0.2)\right.\right.$, \\
& & $\left.\wp_{-1}(0.2), \wp_{1}(0.1)\right\}$, & $\left.\wp_{0}(0.1), \wp_{0}(0.6)\right\}$, & $\left.\wp_{-1}(0.1), \wp_{1}(0.2)\right\}$, & $\left.\wp_{2}(0.6), \wp_{2}(0.1)\right\}$, \\
& & $\left\{\wp_{-1}(0.1), \wp_{-1}(0.5)\right.$, & $\left\{\wp_{-1}(0.5), \wp_{-1}(0.2)\right.$, & $\left\{\wp_{-2}(0.2), \wp_{-1}(0.2)\right.$, & $\left\{\wp_{1}(0.2), \wp_{1}(0.1)\right.$, \\
& & $\left.\left.\wp_{-1}(0.2), \wp_{1}(0.2)\right\}\right\rangle$ & $\left.\left.\wp_{-1}(0.1), \wp_{-1}(0.2)\right\}\right\rangle$ & $\left.\left.\wp_{-1}(0.5), \wp_{1}(0.1)\right\}\right\rangle$ & $\left.\left.\wp_{1}(0.2), \wp_{1}(0.5)\right\}\right\rangle$ \\
\hline
\end{tabular}

Step-3: We calculate the supports $\operatorname{Supp}\left(\tilde{y}_{\wp}^{(i j k)}(\widetilde{\delta}), \tilde{y}_{\wp}^{(i j t)}(\widetilde{\delta})\right)\left(i \in \Lambda_{3} ; j \in \Lambda_{4} ; k, t \in \Lambda_{3} ; k \neq t\right)$ based on Eqs. (13) and (14) and we represent them as $S^{(k t)}\left(k, t \in \Lambda_{3} ; k \neq t\right)$. These are presented in Table-4.

Table-4: Support values

\begin{tabular}{|c|c|c|c|c|}
\hline & $\mathrm{C}_{1}$ & $\mathrm{C}_{2}$ & $\mathrm{C}_{3}$ & $\mathrm{C}_{4}$ \\
\hline \multirow{2}{*}{$\begin{array}{c}S^{(12)} \\
\left(=S^{(21)}\right)\end{array}$} & 0.699537 & 0.298811 & 0.644488 & 0.565387 \\
\cline { 2 - 5 } & 0.621773 & 0.542046 & 0.557469 & 0.528595 \\
\cline { 2 - 5 } & 0.336466 & 0.466406 & 0.483602 & 0.388763 \\
\hline \multirow{2}{*}{$\begin{array}{c}(32) \\
\left(=S^{(23)}\right)\end{array}$} & 0.566987 & 0.841886 & 0.672976 & 0.559041 \\
\cline { 2 - 5 } & 0.427481 & 0.388763 & 0.571826 & 0.634852 \\
\cline { 2 - 5 } & 0.767263 & 0.498613 & 0.62919 & 0.476922 \\
\hline \multirow{2}{*}{$\begin{array}{c}S^{(31)} \\
\left(=S^{(13)}\right)\end{array}$} & 0.49723 & 0.31687 & 0.525658 & 0.616305 \\
\cline { 2 - 5 } & 0.487652 & 0.380861 & 0.566987 & 0.585003 \\
\cline { 2 - 5 } & 0.378622 & 0.726139 & 0.640602 & 0.683772 \\
\hline
\end{tabular}

Step-4: Utilizing Eq. (15), values of $\Theta_{i j k}\left(i \in \Lambda_{3} ; j \in \Lambda_{4} ; k \in \Lambda_{3}\right)$ are obtained (Table-5) by taking $\varpi_{1}=0.32, \varpi_{2}=0.35, \varpi_{3}=0.33$.

Table-5: Values of $\Theta_{i j k}\left(i \in \Lambda_{5} ; j \in \Lambda_{4} ; k \in \Lambda_{3}\right)$

\begin{tabular}{|c|c|c|c|c|}
\hline $\mathbf{D}_{\mathbf{1}}$ & $\mathbf{C}_{\mathbf{1}}$ & $\mathbf{C}_{\mathbf{2}}$ & $\mathbf{C}_{\mathbf{3}}$ & $\mathbf{C}_{\mathbf{4}}$ \\
\hline $\mathrm{A}_{1}$ & 0.33654 & 0.273144 & 0.324569 & 0.336605 \\
\hline $\mathrm{A}_{2}$ & 0.347298 & 0.341951 & 0.332332 & 0.325324 \\
\hline $\mathrm{A}_{3}$ & 0.28754 & 0.343534 & 0.32646 & 0.33982 \\
\hline $\mathbf{D}_{\mathbf{2}}$ & $\mathbf{C}_{\mathbf{1}}$ & $\mathbf{C}_{\mathbf{2}}$ & $\mathbf{C}_{\mathbf{3}}$ & $\mathbf{C}_{\mathbf{4}}$ \\
\hline $\mathrm{A}_{1}$ & 0.347227 & 0.361902 & 0.346602 & 0.32777 \\
\hline $\mathrm{A}_{2}$ & 0.337392 & 0.343356 & 0.333089 & 0.332997 \\
\hline $\mathrm{A}_{3}$ & 0.352696 & 0.307885 & 0.324706 & 0.305904 \\
\hline $\mathbf{D}_{3}$ & $\mathbf{C}_{1}$ & $\mathbf{C}_{2}$ & $\mathbf{C}_{3}$ & $\mathbf{C}_{4}$ \\
\hline $\mathrm{A}_{1}$ & 0.316234 & 0.364955 & 0.32883 & 0.335626 \\
\hline $\mathrm{A}_{2}$ & 0.31531 & 0.314693 & 0.334578 & 0.341679 \\
\hline $\mathrm{A}_{3}$ & 0.359764 & 0.348581 & 0.348834 & 0.354275 \\
\hline
\end{tabular}

Step-5: Utilizing Eq. (16) and taking $\phi=3$, we get the aggregated DPL matrix shown in Table-6.

Table-6: Aggregated DPL matrix

\begin{tabular}{|c|c|c|c|c|}
\hline & $\mathrm{C}_{1}$ & $\mathrm{C}_{2}$ & $\mathrm{C}_{3}$ & $\mathrm{C}_{4}$ \\
\hline & $<\left\{\wp_{-0.502}(0.3)\right.$, & $<\left\{\wp_{0.389}(0.4)\right.$, & $<\left\{\wp_{-1.170}(0.1)\right.$, & $<\left\{\wp_{0.536}(0.2)\right.$, \\
\hline
\end{tabular}




\begin{tabular}{|c|c|c|c|c|}
\hline $\mathrm{A}_{1}$ & $\begin{array}{c}\wp_{1.639}(0.2), \\
\wp_{1.639}(0.2), \\
\left.\wp_{1.683}(0.3)\right\}, \\
\left\{\wp_{-1.890}(0.3),\right. \\
\wp_{-1.882}(0.1), \\
\wp_{-1.692}(0.3), \\
\left.\wp_{-0.842}(0.3)\right\}>\end{array}$ & $\begin{array}{c}\wp_{0.399}(0.2), \\
\wp_{1.587}(0.3), \\
\left.\wp_{1.61}(0.1)\right\}, \\
\left\{\wp_{-1.586}(0.3),\right. \\
\wp_{-1.586}(0.3), \\
\wp_{1.471}(0.1), \\
\left.\wp_{1.471}(0.3)\right\}>\end{array}$ & $\begin{array}{c}\wp_{0.494}(0.3), \\
\wp_{-0.439}(0.2), \\
\left.\wp_{0.846}(0.4)\right\}, \\
\left\{\wp_{0.443}(0.1),\right. \\
\wp_{1.157}(0.3), \\
\wp_{1.157}(0.3), \\
\left.\wp_{1.157}(0.3)\right\}>\end{array}$ & $\begin{array}{c}\wp_{0.536}(0.3), \\
\wp_{1.675}(0.3), \\
\left.\wp_{1.675}(0.2)\right\}, \\
\left\{\wp_{-1.700}(0.3),\right. \\
\wp_{-1}(0.3), \\
\wp_{-0.842}(0.3), \\
\left.\wp_{-0.543}(0.1)\right\}>\end{array}$ \\
\hline $\mathrm{A}_{2}$ & $\begin{array}{c}<\left\{\wp_{-0.475}(0.1),\right. \\
\wp_{1.663}(0.5), \\
\wp_{1.663}(0.2), \\
\left.\wp_{1.664}(0.2)\right\}, \\
\left\{\wp_{-1.694}(0.6),\right. \\
\wp_{-0.828}(0.1), \\
\wp_{-0.828}(0.2), \\
\left.\wp_{1.152}(0.1)\right\}>\end{array}$ & $\begin{array}{c}<\left\{\wp_{1.636}(0.5),\right. \\
\wp_{1.637}(0.2), \\
\wp_{1.660}(0.1), \\
\left.\wp_{1.681}(0.2)\right\}, \\
\left\{\wp_{-1.640}(0.2),\right. \\
\wp_{-0.527}(0.1), \\
\wp_{-0.520}(0.1), \\
\left.\wp_{0.472}(0.6)\right\}>\end{array}$ & $\begin{array}{c}<\left\{\wp_{0.551}(0.2),\right. \\
\wp_{1.679}(0.2), \\
\wp_{1.679}(0.5), \\
\left.\wp_{1.679}(0.1)\right\}, \\
\left\{\wp_{-1.697}(0.1),\right. \\
\wp_{-0.843}(0.6), \\
\wp_{-0.843}(0.1), \\
\left.\wp_{0.433}(0.2)\right\}>\end{array}$ & $\begin{array}{c}<\left\{\wp_{-1.400}(0.2),\right. \\
\wp_{-1.162}(0.1), \\
\wp_{0.550}(0.2), \\
\left.\wp_{0.839}(0.5)\right\}, \\
\left\{\wp_{-1.668}(0.1),\right. \\
\wp_{-1.668}(0.2), \\
\wp_{0.491}(0.6), \\
\left.\wp_{0.491}(0.1)\right\}>\end{array}$ \\
\hline $\mathrm{A}_{3}$ & $\begin{array}{c}<\left\{\wp_{0.423}(0.6),\right. \\
\wp_{0.423}(0.1), \\
\wp_{0.423}(0.2), \\
\left.\wp_{0.806}(0.1)\right\}, \\
\left\{\wp_{-0.869}(0.1),\right. \\
\wp_{-0.846}(0.5), \\
\wp_{-0.846}(0.2), \\
\left.\wp_{1.136}(0.2)\right\}>\end{array}$ & $\begin{array}{c}<\left\{\wp_{1.632}(0.2),\right. \\
\wp_{1.632}(0.1), \\
\wp_{1.635}(0.1), \\
\left.\wp_{1.635}(0.6)\right\}, \\
\left\{\wp_{-1.885}(0.5),\right. \\
\wp_{-1.675}(0.2), \\
\wp_{-1}(0.1), \\
\left.\wp_{-0.812}(0.2)\right\}>\end{array}$ & $\begin{array}{c}<\left\{\wp_{0.474}(0.1),\right. \\
\wp_{0.481}(0.6), \\
\wp_{0.481}(0.1), \\
\left.\wp_{0.824}(0.2)\right\}, \\
\left\{\wp_{-1.894}(0.2),\right. \\
\wp_{-0.847}(0.2), \\
\wp_{-0.847}(0.5), \\
\left.\wp_{0.441}(0.1)\right\}>\end{array}$ & $\begin{array}{c}<\left\{\wp_{1.685}(0.1),\right. \\
\wp_{1.685}(0.2), \\
\wp_{1.895}(0.6), \\
\left.\wp_{1.895}(0.1)\right\}, \\
\left\{\wp_{-1.664}(0.2),\right. \\
\wp_{-1.664}(0.1), \\
\wp_{0.463}(0.2), \\
\left.\wp_{0.463}(0.5)\right\}>\end{array}$ \\
\hline
\end{tabular}

Step-6: Determining criteria weights using FUCOM.

Step 6.1: The ranking of the criteria: $\mathrm{C}_{4}>\mathrm{C}_{1}>\mathrm{C}_{3}>\mathrm{C}_{2}$.

Step 6.2: The comparison was made with respect to the first-ranked $\mathrm{C}_{4}$ criterion and based on the scale $[1,9]$. Thus, the priorities of the criteria $\left(\varpi_{C_{j(k)}}\right)$ for all of the criteria ranked in Step 6.1 were obtained (Table 7).

Table 7: Priorities of criteria

\begin{tabular}{ccccc}
\hline Criteria & $\mathrm{C}_{4}$ & $\mathrm{C}_{1}$ & $\mathrm{C}_{3}$ & $\mathrm{C}_{2}$ \\
\hline$\varpi_{C_{j(k)}}$ & 1 & 1.6 & 2.8 & 4.6
\end{tabular}

Step 6.3: Based on the obtained priorities of the criteria, the comparative priorities of the criteria are calculated and used in the final model for determining the weight coefficients, as follows:

$$
\begin{aligned}
& \min \chi \\
& \text { s.t. }\left\{\begin{array}{l}
\left|\frac{w_{4}}{w_{1}}-1.6\right| \leq \chi,\left|\frac{w_{1}}{w_{3}}-1.75\right| \leq \chi,\left|\frac{w_{3}}{w_{2}}-1.64\right| \leq \chi, \\
\left|\frac{w_{4}}{w_{3}}-2.80\right| \leq \chi,\left|\frac{w_{1}}{w_{2}}-2.88\right| \leq \chi, \\
\sum_{j=1}^{4} w_{j}=1, w_{j} \geq 0, \forall j
\end{array}\right.
\end{aligned}
$$


By solving the problem with Lingo 17.0 software, the final values of the weight coefficients and DFC of the results $\chi=0.00$ are obtained, Table 8 .

Table 8: Criteria weights

\begin{tabular}{ccccc}
\hline Criteria & $w_{1}$ & $w_{2}$ & $w_{3}$ & $w_{4}$ \\
\hline$w_{j}$ & 0.2844 & 0.0988 & 0.1623 & 0.4544
\end{tabular}

Step-7: Using Eqs. (23) and (24), AIDs and IDs are obtained. The EA decision matrix $\wp^{*}$ is given by Table-9.

Table-9: Extended Aggregated DPL matrix

\begin{tabular}{|c|c|c|c|c|}
\hline & $\mathrm{C}_{1}$ & $\mathrm{C}_{2}$ & $\mathrm{C}_{3}$ & $\mathrm{C}_{4}$ \\
\hline AID & $\begin{array}{l}\left\langle\left\{\wp_{-0.502}(0.1)\right\},\right. \\
\left\{\wp_{1.152}(0.6)\right\}>\end{array}$ & $\begin{array}{l}<\left\{\wp_{0.389}(0.1)\right\}, \\
\left\{\wp_{1.471}(0.6)\right\}>\end{array}$ & $\begin{array}{l}\left\langle\left\{\wp_{-1.170}(0.1)\right\},\right. \\
\left\{\wp_{1.157}(0.6)\right\}>\end{array}$ & $\begin{array}{l}\left\langle\left\{\wp_{-1.400}(0.1)\right\},\right. \\
\left\{\wp_{0.491}(0.6)\right\}>\end{array}$ \\
\hline $\mathrm{A}_{1}$ & $\begin{array}{c}<\left\{\wp_{-0.502}(0.3), \wp_{1.639}(0.2),\right. \\
\left.\wp_{1.639}(0.2), \wp_{1.683}(0.3)\right\}, \\
\left\{\wp_{-1.890}(0.3), \wp_{-1.882}(0.1),\right. \\
\left.\wp_{-1.692}(0.3), \wp_{-0.842}(0.3)\right\}>\end{array}$ & $\begin{array}{c}<\left\{\wp_{0.389}(0.4), \wp_{0.399}(0.2),\right. \\
\left.\wp_{1.587}(0.3), \wp_{1.61}(0.1)\right\}, \\
\left\{\wp_{-1.586}(0.3), \wp_{-1.586}(0.3),\right. \\
\left.\wp_{1.471}(0.1), \wp_{1.471}(0.3)\right\}>\end{array}$ & $\begin{array}{l}<\left\{\wp_{-1.170}(0.1), \wp_{0.494}(0.3),\right. \\
\left.\wp_{-0.439}(0.2), \wp_{0.846}(0.4)\right\}, \\
\left\{\wp_{0.443}(0.1), \wp_{1.157}(0.3),\right. \\
\left.\wp_{1.157}(0.3), \wp_{1.157}(0.3)\right\}>\end{array}$ & $\begin{array}{c}<\left\{\wp_{0.536}(0.2), \wp_{0.536}(0.3),\right. \\
\left.\wp_{1.675}(0.3), \wp_{1.675}(0.2)\right\}, \\
\left\{\wp_{-1.700}(0.3), \wp_{-1}(0.3),\right. \\
\left.\wp_{-0.842}(0.3), \wp_{-0.543}(0.1)\right\}>\end{array}$ \\
\hline $\mathrm{A}_{2}$ & $\begin{array}{c}<\left\{\wp_{-0.475}(0.1), \wp_{1.663}(0.5),\right. \\
\left.\wp_{1.663}(0.2), \wp_{1.664}(0.2)\right\}, \\
\left\{\wp_{-1.694}(0.6), \wp_{-0.828}(0.1),\right. \\
\left.\wp_{-0.828}(0.2), \wp_{1.152}(0.1)\right\}>\end{array}$ & $\begin{array}{c}<\left\{\wp_{1.636}(0.5), \wp_{1.637}(0.2),\right. \\
\left.\wp_{1.660}(0.1), \wp_{1.681}(0.2)\right\}, \\
\left\{\wp_{-1.640}(0.2), \wp_{-0.527}(0.1),\right. \\
\left.\wp_{-0.520}(0.1), \wp_{0.472}(0.6)\right\}>\end{array}$ & $\begin{array}{c}<\left\{\wp_{0.551}(0.2), \wp_{1.679}(0.2),\right. \\
\left.\wp_{1.679}(0.5), \wp_{1.679}(0.1)\right\}, \\
\left\{\wp_{-1.697}(0.1), \wp_{-0.843}(0.6),\right. \\
\left.\wp_{-0.843}(0.1), \wp_{0.433}(0.2)\right\}>\end{array}$ & $\begin{array}{c}<\left\{\wp_{-1.400}(0.2), \wp_{-1.162}(0.1),\right. \\
\left.\wp_{0.550}(0.2), \wp_{0.839}(0.5)\right\}, \\
\left\{\wp_{-1.668}(0.1), \wp_{-1.668}(0.2),\right. \\
\left.\wp_{0.491}(0.6), \wp_{0.491}(0.1)\right\}>\end{array}$ \\
\hline $\mathrm{A}_{3}$ & $\begin{array}{c}<\left\{\wp_{0.423}(0.6), \wp_{0.423}(0.1),\right. \\
\left.\wp_{0.423}(0.2), \wp_{0.806}(0.1)\right\}, \\
\left\{\wp_{-0.869}(0.1), \wp_{-0.846}(0.5),\right. \\
\left.\wp_{-0.846}(0.2), \wp_{1.136}(0.2)\right\}>\end{array}$ & $\begin{array}{c}<\left\{\wp_{1.632}(0.2), \wp_{1.632}(0.1),\right. \\
\left.\wp_{1.635}(0.1), \wp_{1.635}(0.6)\right\}, \\
\left\{\wp_{-1.885}(0.5), \wp_{-1.675}(0.2),\right. \\
\left.\wp_{-1}(0.1), \wp_{-0.812}(0.2)\right\}>\end{array}$ & $\begin{array}{c}<\left\{\wp_{0.474}(0.1), \wp_{0.481}(0.6),\right. \\
\left.\wp_{0.481}(0.1), \wp_{0.824}(0.2)\right\}, \\
\left\{\wp_{-1.894}(0.2), \wp_{-0.847}(0.2),\right. \\
\left.\wp_{-0.847}(0.5), \wp_{0.441}(0.1)\right\}>\end{array}$ & $\begin{array}{c}<\left\{\wp_{1.685}(0.1), \wp_{1.685}(0.2),\right. \\
\left.\wp_{1.895}(0.6), \wp_{1.895}(0.1)\right\}, \\
\left\{\wp_{-1.664}(0.2), \wp_{-1.664}(0.1),\right. \\
\left.\left.\wp_{0.463}(0.2), \wp_{0.463}(0.5)\right\}\right\rangle\end{array}$ \\
\hline ID & $\begin{array}{l}\left\{\wp_{1.683}(0.6)\right\}, \\
\\
\left\{\wp_{-1.890}(0.1)\right\}> \\
\end{array}$ & $\begin{array}{l}\left\langle\left\{\wp_{1.681}(0.6)\right\},\right. \\
\left\{\wp_{-1.885}(0.1)\right\}>\end{array}$ & $\begin{array}{l}<\left\{\wp_{1.679}(0.6)\right\}, \\
\left\{\wp_{-1.894}(0.1)\right\}>\end{array}$ & $\begin{array}{l}<\left\{\wp_{1.895}(0.6)\right\} \\
\left\{\wp_{-1.700}(0.1)\right\}>\end{array}$ \\
\hline
\end{tabular}

Step-8: Utilizing Eq. (25) the normalized EA decision matrix $\wp^{{ }^{* N}}=\left[\hat{y}_{\wp}^{(i j)}(\boldsymbol{\mho})\right]_{3 \times 4}$ $=\left[<\left\{\hat{\wp}_{\xi^{i j(u)}}\left(\boldsymbol{\mho}^{(u)}\right)\right\},\left\{\hat{\wp}_{\xi^{i j(v)}}\left(\boldsymbol{\mho}^{(v)}\right)\right\}>\right]_{3 \times 4}$ is obtained and is given in Table 10.

Table-10: Extended normalized Aggregated DPL matrix

\begin{tabular}{|c|c|c|c|c|}
\hline & $\mathrm{C}_{1}$ & $\mathrm{C}_{2}$ & $\mathrm{C}_{3}$ & $\mathrm{C}_{4}$ \\
\hline AID & $\begin{array}{c}<\left\{\wp_{0.199}(0.1)\right\} \\
\quad\left\{\wp_{3}(0.6)\right\}>\end{array}$ & $\begin{array}{c}<\left\{\wp_{1.344}(0.1)\right\}, \\
\left\{\wp_{3}(0.6)\right\}>\end{array}$ & $\begin{array}{c}<\left\{\wp_{-0.654}(0.1)\right\} \\
\quad\left\{\wp_{3}(0.6)\right\}>\end{array}$ & $\begin{array}{c}<\left\{\wp_{-1.040}(0.1)\right\} \\
\left\{\wp_{3}(0.6)\right\}>\end{array}$ \\
\hline $\mathrm{A}_{1}$ & $\begin{array}{c}<\left\{\wp_{0.199}(0.3), \wp_{2.943}(0.2),\right. \\
\left.\wp_{2.943}(0.2), \wp_{3}(0.3)\right\}, \\
\left\{\wp_{-1.396}(0.3), \wp_{-1.384}(0.1),\right. \\
\left.\wp_{-1.111}(0.3), \wp_{0.118}(0.3)\right\}>\end{array}$ & $\begin{array}{c}<\left\{\wp_{1.344}(0.4), \wp_{1.356}(0.2),\right. \\
\left.\wp_{2.878}(0.3), \wp_{2.915}(0.1)\right\}, \\
\left\{\wp_{-1.103}(0.3), \wp_{-1.103}(0.3),\right. \\
\left.\wp_{3}(0.1), \wp_{3}(0.3)\right\}>\end{array}$ & $\begin{array}{c}<\left\{\wp_{-0.654}(0.1), \wp_{1.480}(0.3),\right. \\
\left.\wp_{0.283}(0.2), \wp_{1.931}(0.4)\right\}, \\
\left\{\wp_{1.969}(0.1), \wp_{3}(0.3),\right. \\
\left.\wp_{3}(0.3), \wp_{3}(0.3)\right\}>\end{array}$ & $\begin{array}{c}<\left\{\wp_{1.335}(0.2), \wp_{1.335}(0.3),\right. \\
\left.\wp_{2.730}(0.3), \wp_{2.730}(0.2)\right\}, \\
\left\{\wp_{-0.767}(0.3), \wp_{0.436}(0.3),\right. \\
\left.\wp_{0.707}(0.3), \wp_{1.221}(0.1)\right\}>\end{array}$ \\
\hline $\mathrm{A}_{2}$ & $\begin{array}{l}<\left\{\wp_{0.234}(0.1), \wp_{2.975}(0.5),\right. \\
\left.\wp_{2.975}(0.2), \wp_{2.975}(0.2)\right\}, \\
\left\{\wp_{-1.112}(0.6), \wp_{0.137}(0.1),\right.\end{array}$ & $\begin{array}{c}<\left\{\wp_{2.942}(0.5), \wp_{2.942}(0.2),\right. \\
\left.\wp_{2.972}(0.1), \wp_{3}(0.2)\right\}, \\
\left\{\wp_{-1.175}(0.2), \wp_{0.317}(0.1),\right.\end{array}$ & $\begin{array}{c}<\left\{\wp_{1.553}(0.2), \wp_{3}(0.2),\right. \\
\left.\wp_{3}(0.5), \wp_{3}(0.1)\right\} \\
\left\{\wp_{-1.120}(0.1), \wp_{0.112}(0.6),\right.\end{array}$ & $\begin{array}{c}<\left\{\wp_{-1.040}(0.2), \wp_{-0.747}(0.1),\right. \\
\left.\wp_{1.351}(0.2), \wp_{1.705}(0.5)\right\}, \\
\left\{\wp_{-0.711}(0.1), \wp_{-0.711}(0.2),\right.\end{array}$ \\
\hline
\end{tabular}




\begin{tabular}{|c|c|c|c|c|}
\hline & $\left.\wp_{0.137}(0.2), \wp_{3}(0.1)\right\}>$ & $\left.\wp_{0.326}(0.1), \wp_{1.659}(0.6)\right\}>$ & $\left.\wp_{0.112}(0.1), \wp_{1.954}(0.2)\right\}>$ & $\left.\left.\wp_{3}(0.6), \wp_{3}(0.1)\right\}\right\rangle$ \\
\hline $\mathrm{A}_{3}$ & $\left\langle\wp_{1.386}(0.6), \wp_{1.386}(0.1)\right.$, & $\left\langle\left\{\wp_{2.936}(0.2), \wp_{2.936}(0.1)\right.\right.$, & $\left\langle\left\{\wp_{1.454}(0.1), \wp_{1.464}(0.6)\right.\right.$, & $\left\langle\left\{\wp_{2.743}(0.1), \wp_{2.743}(0.2)\right.\right.$, \\
& $\left.\wp_{1.386}(0.2), \wp_{1.876}(0.1)\right\}$, & $\left.\wp_{2.940}(0.1), \wp_{2.940}(0.6)\right\}$, & $\left.\wp_{1.464}(0.1), \wp_{1.902}(0.2)\right\}$, & $\left.\wp_{3}(0.6), \wp_{3}(0.1)\right\}$, \\
& $\left\{\wp_{0.078}(0.1), \wp_{0.111}(0.5)\right.$, & $\left\{\wp_{-1.504}(0.5), \wp_{-1.223}(0.2)\right.$, & $\left\{\wp_{-1.404}(0.2), \wp_{0.105}(0.2)\right.$, & $\left\{\wp_{-0.704}(0.2), \wp_{-0.704}(0.1)\right.$, \\
& $\left.\left.\wp_{0.111}(0.2), \wp_{2.977}(0.2)\right\}\right\rangle$ & $\left.\left.\wp_{-0.316}(0.1), \wp_{-0.064}(0.2)\right\}\right\rangle$ & $\left.\left.\wp_{0.105}(0.5), \wp_{1.966}(0.1)\right\}\right\rangle$ & $\left.\left.\wp_{2.951}(0.2), \wp_{2.951}(0.5)\right\}\right\rangle$ \\
\hline ID & $\left\langle\left\{\wp_{3}(0.6)\right\}\right.$, & $\left\langle\left\{\wp_{3}(0.6)\right\}\right.$, & $\left\langle\left\{\wp_{3}(0.6)\right\}\right.$, & $\left\langle\left\{\wp_{3}(0.6)\right\}\right.$, \\
& $\left\{\wp_{-1.396}(0.1)\right\}>$ & $\left\{\wp_{-1.504}(0.1)\right\}>$ & $\left\{\wp_{-1.404}(0.1)\right\}>$ & $\left.\left\{\wp_{-0.767}(0.1)\right\}\right\rangle$ \\
\hline
\end{tabular}

Step 9: The constructed weighted normalized EA decision matrix $\wp^{* N W}=\left[\hat{y}_{\wp}^{\prime(i j)}(\boldsymbol{\zeta})\right]_{m \times n}$ is given below (Table-11):

Table-11: Weighted Extended normalized Aggregated DPL matrix

\begin{tabular}{|c|c|c|c|c|}
\hline & $\mathrm{C}_{1}$ & $\mathrm{C}_{2}$ & $\mathrm{C}_{3}$ & $\mathrm{C}_{4}$ \\
\hline AID & $\begin{array}{l}\left\langle\left\{\wp_{-2.090}(0.1)\right\},\right. \\
\left.\left\{\wp_{-1.293}(0.6)\right\}\right\rangle\end{array}$ & $\begin{array}{l}\left\langle\left\{\wp_{-2.571}(0.1)\right\},\right. \\
\left.\left\{\wp_{-2.407}(0.6)\right\}\right\rangle\end{array}$ & $\begin{array}{l}\left\langle\left\{\wp_{-2.619}(0.1)\right\},\right. \\
\left\{\wp_{-2.026}(0.6)\right\}>\end{array}$ & $\begin{array}{l}\left\langle\left\{\wp_{-2.109}(0.1)\right\},\right. \\
\left.\left\{\wp_{-0.273}(0.6)\right\}\right\rangle\end{array}$ \\
\hline $\mathrm{A}_{1}$ & $\begin{array}{l}<\left\{\wp_{-2.090}(0.3), \wp_{-1.309}(0.2),\right. \\
\left.\wp_{-1.309}(0.2), \wp_{-1.293}(0.3)\right\}, \\
\left\{\wp_{-2.544}(0.3), \wp_{-2.540}(0.1),\right. \\
\left.\wp_{-2.462}(0.3), \wp_{-2.113}(0.3)\right\}>\end{array}$ & $\begin{array}{l}<\left\{\wp_{-2.570}(0.4), \wp_{-2.569}(0.2),\right. \\
\left.\wp_{-2.419}(0.3), \wp_{-2.415}(0.1)\right\}, \\
\left\{\wp_{-2.812}(0.3), \wp_{-2.812}(0.3),\right. \\
\left.\wp_{-2.407}(0.1), \wp_{-2.407}(0.3)\right\}>\end{array}$ & $\begin{array}{l}<\left\{\wp_{-2.619}(0.1), \wp_{-2.272}(0.3),\right. \\
\left.\wp_{-2.467}(0.2), \wp_{-2.199}(0.4)\right\}, \\
\left\{\wp_{-2.193}(0.1), \wp_{-2.026}(0.3),\right. \\
\left.\wp_{-2.026}(0.3), \wp_{-2.026}(0.3)\right\}>\end{array}$ & $\begin{array}{l}<\left\{\wp_{-1.030}(0.2), \wp_{-1.030}(0.3),\right. \\
\left.\wp_{-0.396}(0.3), \wp_{-0.396}(0.2)\right\}, \\
\left\{\wp_{-1.985}(0.3), \wp_{-1.438}(0.3),\right. \\
\left.\wp_{-1.315}(0.3), \wp_{-1.081}(0.1)\right\}>\end{array}$ \\
\hline $\mathrm{A}_{2}$ & $\begin{array}{l}<\left\{\wp_{-2.080}(0.1), \wp_{-1.301}(0.5),\right. \\
\left.\wp_{-1.301}(0.2), \wp_{-1.301}(0.2)\right\}, \\
\left\{\wp_{-2.463}(0.6), \wp_{-2.107}(0.1),\right. \\
\left.\wp_{-2.107}(0.2), \wp_{-1.293}(0.1)\right\}>\end{array}$ & $\begin{array}{c}<\left\{\wp_{-2.412}(0.5), \wp_{-2.412}(0.2),\right. \\
\left.\wp_{-2.409}(0.1), \wp_{-2.407}(0.2)\right\}, \\
\left\{\wp_{-2.819}(0.2), \wp_{-2.672}(0.1),\right. \\
\left.\wp_{-2.671}(0.1), \wp_{-2.539}(0.6)\right\}>\end{array}$ & $\begin{array}{c}<\left\{\wp_{-2.261}(0.2), \wp_{-2.026}(0.2),\right. \\
\left.\wp_{-2.026}(0.5), \wp_{-2.026}(0.1)\right\}, \\
\left\{\wp_{-2.695}(0.1), \wp_{-2.494}(0.6),\right. \\
\left.\left.\wp_{-2.494}(0.1), \wp_{-2.195}(0.2)\right\}\right\rangle\end{array}$ & $\begin{array}{l}<\left\{\wp_{-2.109}(0.2), \wp_{-1.976}(0.1),\right. \\
\left.\wp_{-1.022}(0.2), \wp_{-0.861}(0.5)\right\}, \\
\left\{\wp_{-1.960}(0.1), \wp_{-1.960}(0.2),\right. \\
\left.\wp_{-0.273}(0.6), \wp_{-0.273}(0.1)\right\}>\end{array}$ \\
\hline $\mathrm{A}_{3}$ & $\begin{array}{l}<\left\{\wp_{-1.752}(0.6), \wp_{-1.752}(0.1),\right. \\
\left.\wp_{-1.752}(0.2), \wp_{-1.613}(0.1)\right\}, \\
\left\{\wp_{-2.124}(0.1), \wp_{-2.115}(0.5),\right. \\
\left.\wp_{-2.115}(0.2), \wp_{-1.299}(0.2)\right\}>\end{array}$ & $\begin{array}{l}<\left\{\wp_{-2.413}(0.2), \wp_{-2.413}(0.1),\right. \\
\left.\wp_{-2.413}(0.1), \wp_{-2.413}(0.6)\right\}, \\
\left\{\wp_{-2.852}(0.5), \wp_{-2.824}(0.2),\right. \\
\left.\wp_{-2.734}(0.1), \wp_{-2.709}(0.2)\right\}>\end{array}$ & $\begin{array}{l}<\left\{\wp_{-2.277}(0.1), \wp_{-2.275}(0.6),\right. \\
\left.\wp_{-2.275}(0.1), \wp_{-2.204}(0.2)\right\}, \\
\left\{\wp_{-2.741}(0.2), \wp_{-2.495}(0.2),\right. \\
\left.\wp_{-2.495}(0.5), \wp_{-2.193}(0.1)\right\}>\end{array}$ & $\begin{array}{c}<\left\{\wp_{-0.390}(0.1), \wp_{-0.390}(0.2),\right. \\
\left.\wp_{-0.273}(0.6), \wp_{-0.273}(0.1)\right\}, \\
\left\{\wp_{-1.956}(0.2), \wp_{-1.956}(0.1),\right. \\
\left.\wp_{-0.295}(0.2), \wp_{-0.295}(0.5)\right\}>\end{array}$ \\
\hline ID & $\begin{array}{l}<\left\{\wp_{-1.293}(0.6)\right\}, \\
\\
\left\{\wp_{-2.544}(0.1)\right\}>\end{array}$ & $\begin{array}{l}<\left\{\wp_{-2.407}(0.6)\right\}, \\
\\
\left\{\wp_{-2.852}(0.1)\right\}>\end{array}$ & $\begin{array}{l}<\left\{\wp_{-2.026}(0.6)\right\} \\
\\
\left\{\wp_{-2.741}(0.1)\right\}>\end{array}$ & $\begin{array}{l}<\left\{\wp_{-0.273}(0.6)\right\} \\
\\
\left\{\wp_{-1.985}(0.1)\right\}>\end{array}$ \\
\hline
\end{tabular}

Step 10 to 12: Find the utility value of each alternative.

The utility degree of an alternative in relation to AID and ID solution are calculated using Eq. (26) and Eq. (27) respectively. The utility value in relation to the AID and ID solutions are determined using Eq. (28) and (29) respectively. Then, the overall utility value of each alternative is calculated using Eq. (30). All these are depicted in Table-12.

Table-12: Utility values of alternatives

\begin{tabular}{|l|c|c|c|c|c|}
\hline & $\hbar_{i}^{-}$ & $\hbar_{i}^{+}$ & $\lambda_{i}^{-}$ & $\lambda_{i}^{+}$ & $\lambda_{i}$ \\
\hline $\mathrm{A}_{1}$ & -0.45968675 & 0.4498061 & -45.52393973 & 46.52393973 & 0.009875987 \\
\hline $\mathrm{A}_{2}$ & -0.29294335 & 0.28664673 & -45.52393973 & 46.52393973 & 0.006293644 \\
\hline $\mathrm{A}_{3}$ & -0.40329193 & 0.39462345 & -45.52393973 & 46.52393973 & 0.008664391 \\
\hline
\end{tabular}

Step 13: The alternatives are ranked based on their final utility value. 
Thus the final ranking order is: $A_{1} \succ A_{3} \succ A_{2}$ where " $\succ$ " means "superior to". Hence, the most suitable alternative is $A_{1}$.

\section{Discussion:}

This segment is divided into three sub-sections: (1) the impact in the positioning of options when occurring the changes in the parameter $(\phi),(2)$ a sensitivity examination of the developed method considering various arrangements of criteria weight values; (3) a comparative investigation between the developed MCGDM approach and the related existing methodologies. The subtleties of these three sub-sections are discussed below.

\subsection{Affect of the parameter ' $\phi$ ' on utility values and ranking order}

To investigate the impact of parameters ' $\phi$ ' upon overall utility values and ranking order, we deploy the operator DPLDPWAA for different values of $\phi$ lying in the range $[1,10]$ and next we summarize the final utility values that are reflected in Fig.2 given below.

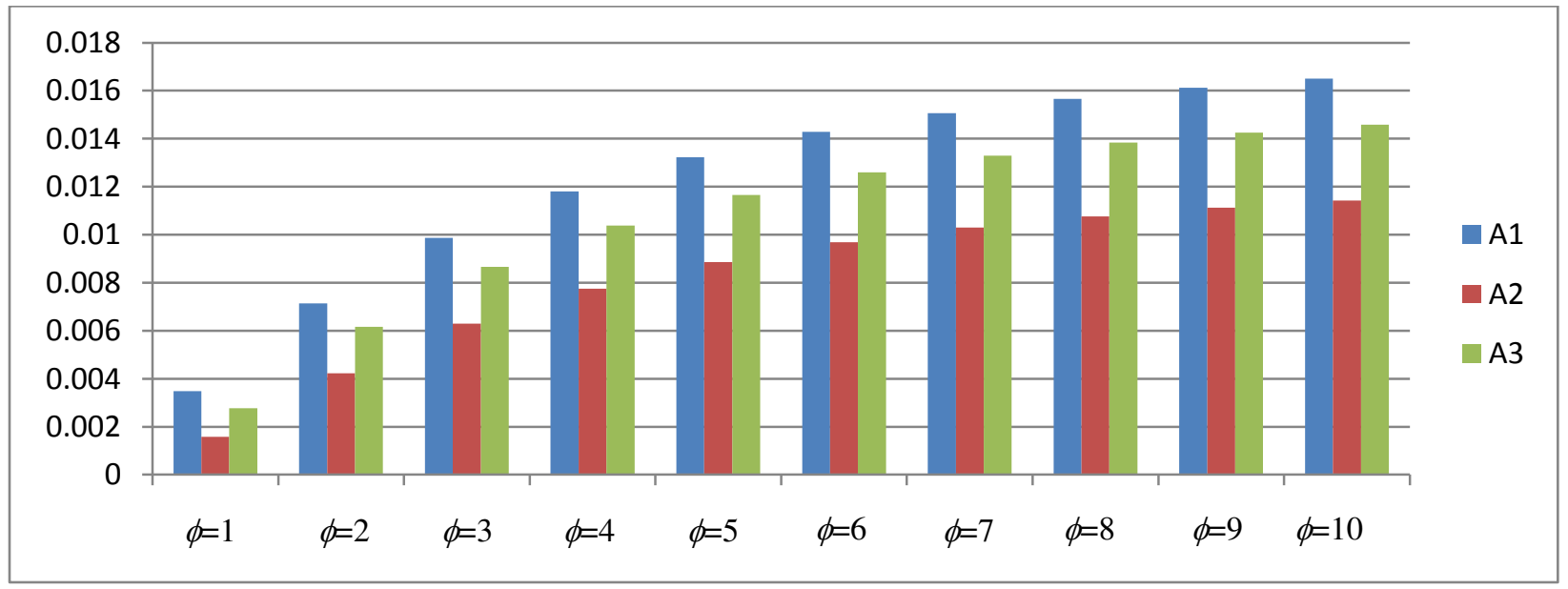

Fig. 2: Overall utility values of the alternatives obtained by DPLDPWAA operator for different $\phi$

From Fig. 2, we observe the fact of increasing nature of the overall utility values of $A_{1}, A_{2}$ and $A_{3}$ deduced from DPLDPWAA operator with the increase of $\phi$ lying in between 1 to 10 .

As discussed above, the utility of the alternatives are computed for various parameter $(\phi)$ values lying in $[1,10]$ and are portrayed in Fig. 2. Their corresponding ranking positions are presented in Table-13. Analyzing the ranking position of the alternatives for different values of $\phi$ in $[1,10]$, we can conclude that $A_{1}$ is $1^{\text {st }}$ ranked in all the cases, $A_{2}$ is $3^{\text {rd }}$ ranked in all the cases and $A_{3}$ is $2^{\text {nd }}$ ranked in all the cases. These investigation shows that the alternative $A_{1}$ is more acceptable compare to all other alternatives. Next, we have computed the SRCC values (Ghorabaee et al. 2016) for different values of $\phi$ in $[1,10]$ that elicited in Table 13. We notice in Table 13 that the average of the SRCC values is 1.00, which shows a "strong correlation" (Ghorabaee et al. 2016) of ranks of alternatives. Thus, with the results, it can be concluded that the rank of the alternatives derived using our proposed method are credible and reliable. In other words, the parameter $\phi$ do not show any sensitive nature during our developed MCGDM process. 
Table-13: Rank of the alternatives for various values of the parameter ' $\phi$ '

\begin{tabular}{|l|c|c|c|c|c|c|c|c|c|c|}
\hline & $\phi=1$ & $\phi=2$ & $\phi=3$ & $\phi=4$ & $\phi=5$ & $\phi=6$ & $\phi=7$ & $\phi=8$ & $\phi=9$ & $\phi=10$ \\
\hline $\mathrm{A}_{1}$ & 1 & 1 & 1 & 1 & 1 & 1 & 1 & 1 & 1 & 1 \\
\hline $\mathrm{A}_{2}$ & 3 & 3 & 3 & 3 & 3 & 3 & 3 & 3 & 3 & 3 \\
\hline $\mathrm{A}_{3}$ & 2 & 2 & 2 & 2 & 2 & 2 & 2 & 2 & 2 & 2 \\
\hline SRCC & 1 & 1 & 1 & 1 & 1 & 1 & 1 & 1 & 1 & 1 \\
\hline
\end{tabular}

\section{2 Sensitivity assessment (SA)}

Here we enforce sensitivity assessment (SA) to evaluate the influence of an attribute on the results of introduced model. This analysis is based on 24 criteria weights sets, namely- WST1, WST2, ......, WST24 (Table-14). Actually, these weight sets are formed by permutation of the original weight set $\{0.2844,0.0988,0.1623,0.4544\}$. This is very much useful to have a more extensive range of attribute weights for looking at the affectability of the developed framework. We compile the overall utility values of the alternative that are reflected in Fig.3. Their corresponding ranking positions are depicted in Table-15. Scrutinizing the ranking position of the $1^{\text {st }}$ alternative $\left(A_{1}\right)$, we can conclude that $A_{1}$ is $1^{\text {st }}$ ranked in $50 \%$ cases, $2^{\text {nd }}$ ranked in $25 \%$ cases and $3^{\text {rd }}$ ranked in the remaining $25 \%$ cases. $A_{2}$ is $1^{\text {st }}$ ranked in $12.5 \%$ cases, $2^{\text {nd }}$ ranked in $25 \%$ cases and $3^{\text {rd }}$ ranked in remaining $62.5 \%$ cases. $A_{3}$ is $1^{\text {st }}$ ranked in $37.5 \%$ cases, $2^{\text {nd }}$ ranked in $50 \%$ cases and $3^{\text {rd }}$ ranked in the remaining $12.5 \%$ cases. These investigation shows that the alternative $A_{1}$ is more acceptable compare to all other alternatives. Thus, with the results, we can say that the rank of the alternatives obtained by using our developed methodology is credible and reliable.

Table-14: Criteria weight sets

\begin{tabular}{|l|c|c|c|c|c|c|c|c|c|c|c|c|}
\hline & WST1 & WST2 & WST3 & WST4 & WST5 & WST6 & WST7 & WST8 & WST9 & WST10 & WST11 & WST12 \\
\hline $\mathrm{C}_{1}$ & 0.2844 & 0.2844 & 0.2844 & 0.2844 & 0.2844 & 0.2844 & 0.0988 & 0.0988 & 0.4544 & 0.4544 & 0.1623 & 0.1623 \\
\hline $\mathrm{C}_{2}$ & 0.0988 & 0.0988 & 0.4544 & 0.4544 & 0.1623 & 0.1623 & 0.2844 & 0.2844 & 0.2844 & 0.2844 & 0.2844 & 0.2844 \\
\hline $\mathrm{C}_{3}$ & 0.1623 & 0.4544 & 0.0988 & 0.1623 & 0.4544 & 0.0988 & 0.1623 & 0.4544 & 0.1623 & 0.0988 & 0.4544 & 0.0988 \\
\hline $\mathrm{C}_{4}$ & 0.4544 & 0.1623 & 0.1623 & 0.0988 & 0.0988 & 0.4544 & 0.4544 & 0.1623 & 0.0988 & 0.1623 & 0.0988 & 0.4544 \\
\hline & WST13 & WST14 & WST15 & WST16 & WST17 & WST18 & WST19 & WST20 & WST21 & WST22 & WST23 & WST24 \\
\hline $\mathrm{C}_{1}$ & 0.1623 & 0.0988 & 0.0988 & 0.4544 & 0.1623 & 0.4544 & 0.1623 & 0.0988 & 0.0988 & 0.4544 & 0.1623 & 0.4544 \\
\hline $\mathrm{C}_{2}$ & 0.0988 & 0.1623 & 0.4544 & 0.0988 & 0.4544 & 0.1623 & 0.0988 & 0.1623 & 0.4544 & 0.0988 & 0.4544 & 0.1623 \\
\hline $\mathrm{C}_{3}$ & 0.2844 & 0.2844 & 0.2844 & 0.2844 & 0.2844 & 0.2844 & 0.4544 & 0.4544 & 0.1623 & 0.1623 & 0.0988 & 0.0988 \\
\hline $\mathrm{C}_{4}$ & 0.4544 & 0.4544 & 0.1623 & 0.1623 & 0.0988 & 0.0988 & 0.2844 & 0.2844 & 0.2844 & 0.2844 & 0.2844 & 0.2844 \\
\hline
\end{tabular}

Table 15: Ranking of the alternatives with various criteria weight sets

\begin{tabular}{|c|c|c|c|c|c|c|c|c|c|c|c|c|}
\hline & WST1 & WST2 & WST3 & WST4 & WST5 & WST6 & WST7 & WST8 & WST9 & WST10 & WST11 & WST12 \\
\hline $\mathrm{A}_{1}$ & 1 & 1 & 1 & 3 & 3 & 1 & 2 & 1 & 2 & 1 & 1 & 2 \\
\hline $\mathrm{A}_{2}$ & 3 & 3 & 3 & 2 & 1 & 3 & 3 & 3 & 1 & 3 & 3 & 3 \\
\hline $\mathrm{A}_{3}$ & 2 & 2 & 2 & 1 & 2 & 2 & 1 & 2 & 3 & 2 & 2 & 1 \\
\hline & WST13 & WST14 & WST15 & WST16 & WST17 & WST18 & WST19 & WST20 & WST21 & WST22 & WST23 & WST24 \\
\hline $\mathrm{A}_{1}$ & 2 & 2 & 3 & 1 & 3 & 3 & 1 & 1 & 3 & 1 & 2 & 1 \\
\hline $\mathrm{A}_{2}$ & 3 & 3 & 2 & 3 & 2 & 1 & 3 & 3 & 2 & 2 & 3 & 2 \\
\hline $\mathrm{A}_{3}$ & 1 & 1 & 1 & 2 & 1 & 2 & 2 & 2 & 1 & 3 & 1 & 3 \\
\hline
\end{tabular}




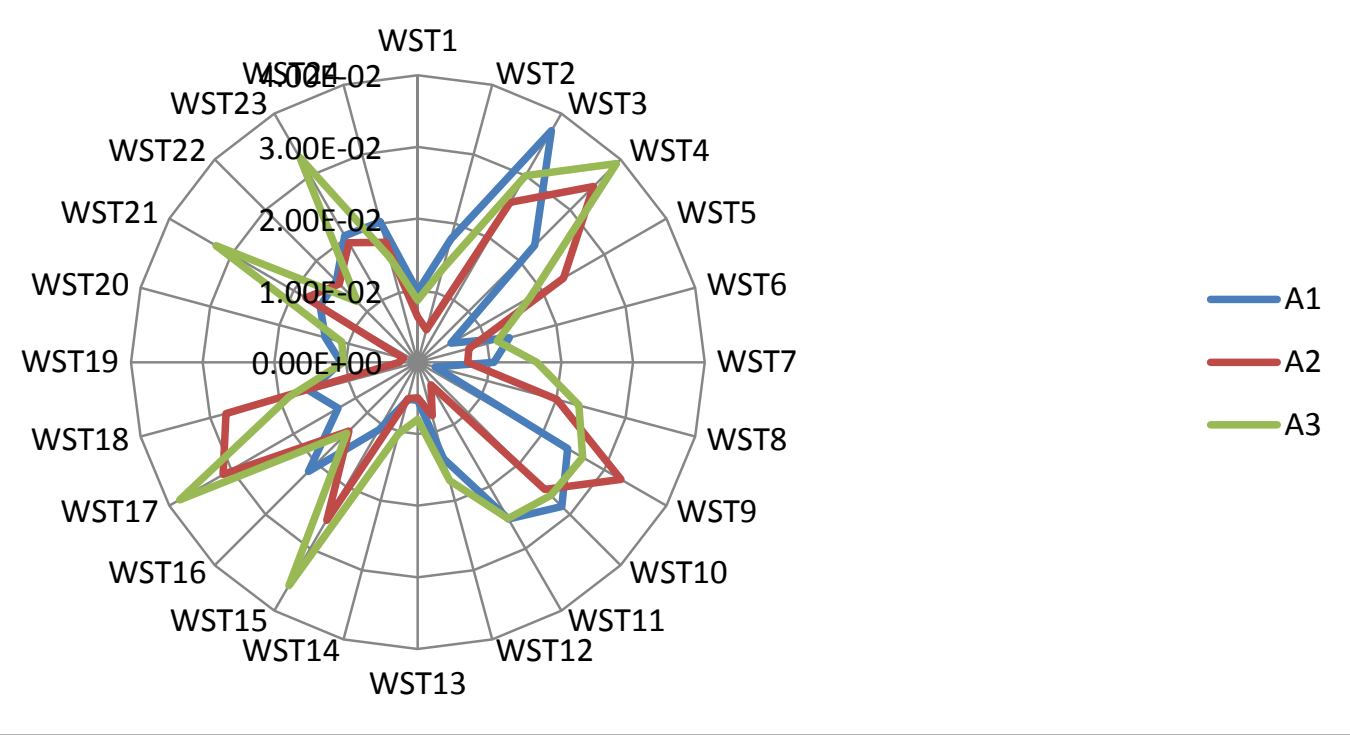

Fig.3: Overall utility values of the alternatives for various attribute weight sets

\subsection{Comparative investigation: Proposed vs. existing}

This section put forwards a comparative investigation from both theoretical and numerical perspectives. To compare our developed MCGDM method presented in section 5 with some existing MCGDM techniques under the DPL settings, we conducted an analysis with some of the existing methods, namely- Xie et al. (2017), and Xie et al. (2020) method.

To illustrate the effectiveness of the developed approach, we apply Xie et al. (2017), and Xie et al. (2020) method to the same case study discussed earlier. The results are summarized in Table 16.

Table-16: Comparative study: Proposed vs existing

\begin{tabular}{|l|c|c|c|}
\multicolumn{1}{|c|}{ Factors } & Proposed & Xie et al. (2017) & Xie et al. (2020) \\
\hline Information type & DPL & DPL & DPL \\
\hline Decision-making type & Group decision-making & $\begin{array}{c}\text { Group decision- } \\
\text { making }\end{array}$ & $\begin{array}{c}\text { Group decision- } \\
\text { making }\end{array}$ \\
\hline $\begin{array}{l}\text { Criteria weights calculation } \\
\text { Priority relation between } \\
\text { criteria }\end{array}$ & FUCOM & Direct & $\begin{array}{c}\text { Optimization } \\
\text { technique }\end{array}$ \\
\hline Aggregation tool & $\begin{array}{c}\text { Considered } \\
\left(\mathrm{C}_{4}>\mathrm{C}_{1}>\mathrm{C}_{3}>\mathrm{C}_{2}\right)\end{array}$ & Not considered & Not considered \\
\hline $\begin{array}{l}\text { Flexibility of the } \\
\text { Aggregation operators }\end{array}$ & AOs & AOs & Preference relations \\
\hline $\begin{array}{l}\text { Whether captures hesitation } \\
\text { in preferences }\end{array}$ & Very high & Very low & NA \\
\hline $\begin{array}{l}\text { Whether deals with } \\
\text { probabilistic information }\end{array}$ & Yes & Yes & Yes \\
\hline $\begin{array}{l}\text { Whether probabilities are in } \\
\text { adjusted form }\end{array}$ & Yes & Yes & Yes \\
\hline
\end{tabular}




\begin{tabular}{|l|c|c|c|}
$\begin{array}{l}\text { Whether diminishes the } \\
\text { impacts of outrageous } \\
\text { assessing information from } \\
\text { some biased DEs }\end{array}$ & Yes & No & No \\
\hline $\begin{array}{l}\text { Non-membership } \\
\text { belongingness degree }\end{array}$ & Considered & Considered & Considered \\
\hline Applied Model & MARCOS & Nil & Nil \\
\hline $\begin{array}{l}\text { Sensitivity of criteria } \\
\text { weights }\end{array}$ & Investigated & Not investigated & Not investigated \\
\hline Ranking of alternatives & $A_{1} \succ A_{3} \succ A_{2}$ & $A_{1} \succ A_{3} \succ A_{2}$ & $A_{1} \succ A_{2} \succ A_{3}$ \\
\hline
\end{tabular}

Table 16 clearly demonstrates the strengths of our proposed method. From analysis we can infer the following:

1. We have used DPL based information which is a powerful form to handle uncertain data representation. Actually, DPLTSs improves consistency and flexibility of the traditional DM methods by considering non-belongingness grades and the simultaneous occurrence of stochastic and non-stochastic uncertainty in real life problems. Thus, DPLTSs are superior to PLTSs.

2. The proposed AOs are based on reversible transformation functions (RTFs). The advantage of RFT is that it can be applied to represent the semantics of RFT which assign the semantic to LTs under different environments.

3. To avoid the circumstances of unreasonable output in aggregation process, we have used the concept of adjusted DPLEs.

4. The DPL-Dombi Power weighted average and geometric AOs can effectively diminish the impacts of outrageous assessing information from some biased DEs during the DM process. Thus our method improves the rationality of the DM process.

5. The DPL-Dombi Power weighted average and geometric AOs can effectively aggregate the DPL information with higher flexibility for the reason that Dombi operators contain a parameter ' $\phi$ ' values of which can be chosen according to real decision needs. Thus, our methodology discussed in this work can be treated as one of the best effective tools developed so far to tackle the MCGDM problems under DPL environment.

6. Our proposed method used the FUCOM technique to determine criteria weights. In contrast to other subjective models (the AHP, the BWM and others), FUCOM displayed smaller deviations of the achieved degrees of the criteria from the most favorable values (Pamucar et al. 2018a). Thus, our method reduces inaccuracies in the MCGDM process.

7. For rational aggregation of preferences, we have used MARCOS method which provides a robust decision making by (i) defining ideal and anti-ideal values (reference points), (ii) determining the connection among alternatives and ideal/anti-ideal values, (iii) characterizing the utility degree of each alternative in connection to ideal and anti-ideal solution. Accordingly, the outcomes acquired by the MARCOS method are more sensible because of the combination of the ratio approach and reference point sorting approach.

8. The sensitivity investigation of the parameter confirms that the developed model is credible and consistent. Since the SRCC value is "1", the proposed approach is steady even after ample variations are used on attribute weights. 


\section{Conclusion:}

Our aim in this paper was to deal with uncertainty and impreciseness associated with decisionmaking problems by using DPLTSs which are generalized from of PLTSs. The existing tools developed so far, for aggregating DPL information, have restricted themselves to algebraic operators, and yet lacked flexibility. This motivated us to use Dombi operations, in introducing some novel operations between DPLEs. The prominent characteristics of these developed operations are analyzed. Furthermore, using the concept of power AOs, we also designed two AOs namely- DPLDPWAA, and DPLDPWGA operators. Some crucial characteristics such as idempotency, boundedness, monotonicity etc., of the developed AOs are discussed in detail. Moreover, depending on them, a FUCOMMARCOS based MCGDM methodology is demonstrated to find the most preferable alternative in DPL environment. In this proposed methodology, criteria weights are calculated using FUCOM technique. To provide a better understanding of our method, we have included a case study involving OSS-LMS selection, and through sensitivity investigation of criteria weights, we have proved the robustness of the proposed methodology. The comparative study enables us to suggest that the developed methodology can be effectively used in MCGDM problems in DPL setting.

The following managerial implications are obtained in this research:

(i) The developed methodology supports the DPL theory to provide a decision framework that fuses imprecise judgments inalienable in the OSS-LMS selection process.

(ii) This paper improves the theoretical base of DPLTSs by proposing new operational laws, aggregation operators, and realizing their crucial properties analytically.

(iii) The combination of power operator with Dombi t-norms and t-conorms mitigates the effect of preference spikes from experts and the methodically calculation of weights of DEs and criteria reduces inaccuracies and biases in the decision system.

(iv) The sensitivity examination and comparative investigation show that the preference orders of the alternatives by the proposed method are reliable with the other existing MCGDM techniques. Henceforth, the proposed structure is productive to catch decision makers' judgments in SSS issue.

In the future, other families of t-norms and t-conorms can be formulated with DPLTSs, new decision models with integrated approaches can be developed for providing practical solution to decision problems, namely- charging station's site selection for electric vehicle, treatment technology selection for medical waste, technological forecasting method selection, cloud vendor selection problem etc. Further, information measures for DPLTSs can be developed for determination of DEs weights.

\section{Compliance with ethical standards:}

Conflict of interest: The authors declare that they do not have any conflict of interest.

Ethical approval: This article does not contain any studies with human participants or animals performed by any of the authors.

\section{Authorship contributions:}

Conceptualization: Abhijit Saha, Methodolody: Abhijit Saha and Arunodaya Raj Mishra, Formal analysis and investigation: Arunodaya Raj Mishra and Pratibha Rani, Writing original draft: Abhijit Saha, Review and editing: Arunodaya Raj Mishra and Pratibha Rani. 


\section{References}

Akram M, Dudek WA, Dar JM (2019) Pythagorean Dombi fuzzy aggregation operators with application in multicriteria decision-making. Int J of Intell Syst 34(11): 3000-3019.

Ashraf S, Abdullah S, Mahmood T (2020) Spherical fuzzy Dombi aggregation operators and their application in group decision making problems. $\mathrm{J}$ of Ambient Intell and Humanized Comput 11: 2731-2749.

Badi I, Pamučar D (2020) Supplier Selection For Steelmaking Company By Using Combined GREYMARCOS Methods. Decis Mak: Appl in Manag and Engg 3(2): 37-47.

Badi I, Abdulshahed A (2019) Ranking the Libyan airlines by using full consistency method (FUCOM) and analytical hierarchy process (AHP). Ope Res in Engg Sci: Theory and Appl. 2(1): 114.

Dombi J (1982) A general class of fuzzy operators, the De Morgan class of fuzzy operators and fuzziness measures induced by fuzzy operators. Fuzzy Sets and Syst 8: 149-163.

Fazlollahtabar H, Smailbasic A, Stevic Z (2019) FUCOM method in group decision-making: Selection of forklift in a warehouse. Decis Mak: Appl in Manag and Engg 2(1): 49-65.

Ghorabaee MK, Zavadskas EK, Amiri M, Esmaeili A (2016) Multi-criteria evaluation of green suppliers using an extended WASPAS method with interval type-2 fuzzy sets. J Cleaner Prod 137: 213-229.

Gou XJ, Xu ZS, Liao HC (2017) Multi-criteria decision making based on Bonferroni means with hesitant fuzzy linguistic information. Soft Comput 21: 6515-6529.

He X (2018) Typhoon disaster assessment based on Dombi hesitant fuzzy information aggregation operators. Nat Hazards 90(3): 1153-1175.

Herrera F, Martinez L (2000) A 2-tuple fuzzy linguistic representation model for computing with words. IEEE Trans Fuzzy Syst 8: 746-752.

Jana C, Senapati T, Pal M, Yager RR (2019a) Picture fuzzy Dombi aggregation operators: application to MADM process. Appl Soft Comput 74: 99-109.

Jana C, Muhiuddin G, Pal M (2019b) Some Dombi aggregation of q-rung orthopair fuzzy numbers in multiple-attribute decision making. Int J of Intell Syst 34(12):3220-3240.

Jana C, Pal M, Wang JQ (2019c) Bipolar fuzzy Dombi aggregation operators and its application in multiple-attribute decision-making process. J of Ambient Intell and Humanized Comput 10: 35333549.

Jana C, Pal M, Wang JQ (2020) Bipolar fuzzy Dombi prioritized aggregation operators in multiple attribute decision making. Soft Comput 24: 3631-3646.

Kobina A, Liang D, He X (2017) Probabilistic linguistic power aggregation operators for multi-criteria group decision making. Symmetry 9(12): 1-21.

Krishankumar R, Ravichandran KS, Ahmed M, Kar S, Tyagi S (2018) Probabilistic Linguistic Preference Relation-Based Decision Framework for Multi-Attribute Group Decision Making. Symmetry 11(1): 1-18.

Krishankumar R, Saranya R, Nethra RP, Ravichandran KS, Kar S (2019) A decision-making framework under probabilistic linguistic term set for multi-criteria group decision-making problem. J Intell Fuzzy Syst 36(6): 5783-5795.

Li B, Xu Zs, Zhang YX (2020) A probabilistic linguistic evaluation based multi-stage medical system selection process related to referral system. Expert Syst with Appl 170: 114523 (https:/doi.org/10.1016/j.eswa.2020.114523).

Liao H, Jiang L, Xu Z, Xu J, Herrera F (2017) A linear programming method for multiple criteria decision making with probabilistic linguistic information. Inform Sci 416: 341-355.

Liu P, Teng F (2019) Probabilistic linguistic TODIM method for selecting products through online product reviews. Inform Sci 485: 441-455. 
Liu P, Liu JL, Chen SM (2018) Some intuitionistic fuzzy Dombi Bonferroni mean operators and their application to multi-attribute group decision making. J Oper Res Soc 69(1): 1-24.

Liu HB, Liu Y, Xu L (2020) Dombi Interval-Valued Hesitant Fuzzy Aggregation Operators for Information Security Risk Assessment. Math Prob in Engg. (Article ID- 3198645), 01-15.

Liao H, Mi X, Xu Z (2020) A survey of decision-making methods with probabilistic linguistic information : Bibliometrics, preliminaries, methodologies, applications and future directions. Fuzzy Opt and Dec Mak 19: 81-134.

Liao H, Wu X (2020) DNMA: A double normalization-based multiple aggregation method for multi-expert multi-criteria decision making. Omega (http://doi.org/10.1016/j.omega.2019.04.001).

Noureddine M, Ristic M (2019) Route planning for hazardous materials transportation: Multi-criteria decision making approach. Decis Mak: Appl in Manag and Engg 2(1): 66-85.

Pamucar D, Stevic Z, Sremac S (2018a) A New Model for Determining Weight Coefficients of Criteria in MCDM Models: Full Consistency Method (FUCOM). Symmetry 10: 393.

Pamucar D, Lukovac V, Bozanic D, Komazec N (2018b) Multi-criteria FUCOM-MAIRCA model for the evaluation of level crossings: case study in the Republic of Serbia. Ope Res in Engg Sci: Theory and Appl 1(1): 108-129.

Pamucar D, Deveci M, Canitez F, Bozanic D (2019) A fuzzy Full Consistency Method-DombiBonferroni model for priorititizing transportation demand management measures. Appl Soft Comput (doi: https://doi.org/10.1016/j.asoc.2019.105952).

Pang Q, Wang H, Xu ZS (2016) Probabilistic Linguistic Term Sets in Multi-Attribute Group Decision Making. Inform Sci 369: 128-143.

Qiyas M, Abdullah S, Ashraf S, Abdullah L (2019) Linguistic Picture Fuzzy Dombi Aggregation Operators and Their Application in Multiple Attribute Group Decision-Making problem. Mathematics 7: 01-23.

Ramadass S, Krishankumar R, Ravichandran KS, Liao H, Kar S (2020) Evaluation of cloud vendors from probabilistic linguistic information with unknown/partial weight values. Appl Soft Comput 97:106801.

Rodríguez RM, Martínez L, Herrera F (2012) Hesitant Fuzzy Linguistic Term Sets for Decision Making. IEEE Trans Fuzzy Syst 20: 109-119.

Seikh MR, Mandal U (2019) Intuitionistic fuzzy Dombi aggregation operators and their application to multiple attribute decision-making. Granular Comput (https://doi.org/10.1007/s41066-019-00209-y).

Shi L, Ye J (2018) Dombi aggregation operators of neutrosophic cubic sets for multiple attribute decision-making. Algorithms. 11(3): 29 (https://doi.org/10.3390/a11030029).

Stanković M, Stević Ž, Das DK, Subotic M, Pamučar D (2020) A New Fuzzy MARCOS Method for Road Traffic Risk Analysis. Mathematics 8: 01-18.

Stevic Z, Pamucar D, Puska A, Chatterjee P (2019) Sustainable supplier selection in healthcare industries using a new MCDM method: Measurement Alternatives and Ranking according to compromise solution (MARCOS). Comput and Indus Engg (https://doi.org/10.1016/ j.cie. 2019.106231).

Stević Ž, Brković N (2020) A Novel Integrated FUCOM-MARCOS Model for Evaluation of Human Resources in a Transport Company. Logistics 4: 01-14.

Ulutaş A, Karabasevic D, Popovic G, Stanujkic D, Nguyen PT, Karaköy Ç (2020) Development of a Novel Integrated CCSD-ITARA-MARCOS Decision-Making Approach for Stackers Selection in a Logistics System. Mathematics 8: 01-15.

Wang XX, Xu ZS, wen Q, Li H (2021) A multi-dimensional decision with nested probabilistic linguistic term sets and its application in corporate investment. Econo Res Ekonomska Istrazivanja (https://doi.org/10.1080/1331677X.2021.1875255).

Wei G, Wei C, Wu J, Guo Y (2021) Probabilistic linguistic multiple attribute group decision making 
for location planning of electric vehicle charging stations based on the generalized Dice similarity measures. Artifi Intell Rev (https://doi.org/10.1007/s10462-020-09950-2).

Wei G, Wei Y (2018) Some single-valued neutrosophic Dombi prioritized weighted aggregation operators in multiple attribute decision making. J of Intell and Fuzzy Syst 35(2), 2001-2013.

Wu L, Wei G, Wu J, Wei C (2020) Some Interval-Valued Intuitionistic Fuzzy Dombi Heronian Mean Operators and their Application for Evaluating the Ecological Value of Forest Ecological Tourism Demonstration Areas. Int J of Environ Res and Pub Health 17: 01-31.

Xie W, Xu ZS, Ren Z (2017) Dual probabilistic linguistic term set and its application on the multicriteria group decision-making problems, in: 2017 IEEE International Conference on Industrial Engg. And Engg. Management, IEEE, 2017, pp. 1469-1474.

Xie W, Xu ZS, Ren Z, Viedma EH (2020) A new multi-criteria decision-making model based on incomplete dual probabilistic linguistic preference relations. Appl Soft Comput 91: 106237.

Xu GL, Wan SP, Dong JY (2020) An Entropy-Based Method for Probabilistic Linguistic Group Decision-Making and its Application of Selecting Car Sharing Platforms. Informatica 31(3): 621658.

Xue WT, Zu ZS, Mi X, Ren ZL (2021) Dynamic reference point method with probabilistic linguistic information based on the regret theory for public health emergency decision-making. Econo Res Ekonomska Istrazivanja (http://doi.org/10.1080/1331677X.2021.1875254).

Yager RR (2001) The power average operator. IEEE Trans Syst Man Cybern 31(6): 724-731.

Yazdani M, Chatterjee P, Pamucar D, Chakrabort, S (2020) Development of an integrated decision making model for location selection of logistics centers in the Spanish autonomous communities, Expert Syst With Appl (https://doi.org/10.1016/ j.eswa.2020. 113208).

Zadel LA (1975) The concept of a linguistic variable and its application to approximate reasoning-I. Inform Sci 8(3): 199-249.

Zhang X, Xing X (2017) Probabilistic Linguistic VIKOR Method to Evaluate Green Supply Chain Initiatives. Sustainability 9(7): 1-18. 


\section{Supplementary Files}

This is a list of supplementary files associated with this preprint. Click to download.

- SUPPLEMENTARYDOMBIDPL.pdf 